\title{
BAIRE REDUCTIONS AND GOOD BOREL REDUCIBILITIES
}

\author{
LUCA MOTTO ROS
}

\begin{abstract}
In [8] we have considered a wide class of "well-behaved" reducibilities for sets of reals. In this paper we continue with the study of Borel reducibilities by proving a dichotomy theorem for the degree-structures induced by good Borel reducibilities. This extends and improves the results of [8] allowing to deal with a larger class of notions of reduction (including, among others, the Baire class $\xi$ functions).
\end{abstract}

\section{INTRODUCTION}

A reducibility for sets of reals ${ }^{1}$ is simply a collection $\mathcal{F}$ of functions from $\mathbb{R}$ to $\mathbb{R}$ which is used to reduce a set of reals to another one: given $A, B \subseteq \mathbb{R}$, we say that $A$ is $\mathcal{F}$-reducible to $B$ just in case there is some $f \in \mathcal{F}$ such that $x \in A \Longleftrightarrow f(x) \in B$ for every $x \in \mathbb{R}$. Such an $\mathcal{F}$ allows to measure the "relative complexity" of the sets of reals, and $\mathcal{F}$ itself can be viewed as the "unit of measurement" that we are using: in general, the "smaller" is our set $\mathcal{F}$, the more accurate is our measurement (i.e. the finer is the hierarchy of degrees induced by $\mathcal{F}$ ).

The first two reducibilities that one encounters in the literature are the collection W of all continuous functions and the collection $L$ of all Lipschitz functions with constant less than or equal to 1 . The corresponding degree-structures were extensively studied (assuming AD, the Axiom of Determinacy) by Wadge, Steel, Van Wesep and many other set theorists (Martin, Kechris, Louveau, Saint-Raymond to name a few), and have had many applications in Set Theory and Theoretical Computer Science (see for example [11] or [5]). Some years ago, Andretta and Martin considered the collection Bor of all Borel functions and the collection $\mathrm{D}_{2}$ of all $\boldsymbol{\Delta}_{2}^{0}$-functions, and they proved that in both cases the degree-structures induced by those reducibilities look like the Wadge one, i.e. like the one induced by continuous functions. In [8] we have described a general method to extend this analysis to the so-called Borel-amenable reducibilities, among which there are e.g. the continuous functions, the Borel functions, the collection $\mathrm{D}_{\xi}$ of all $\boldsymbol{\Delta}_{\xi}^{0}$-functions for $\xi<\omega_{1}$, i.e. the collection of those $f$ such that $f^{-1}(D) \in \boldsymbol{\Delta}_{\xi}^{0}$ for every $D \in \boldsymbol{\Delta}_{\xi}^{0}$, and so on. As for the previous cases, we have obtained that whenever $\mathcal{F}$ is a Borel-amenable set of reductions the degree-structure induced by $\mathcal{F}$ looks like the Wadge one.

Since the $D_{\xi}$ 's form a natural stratification of the Borel functions, the present work was mainly motivated by the natural idea of considering the other classical stratification of the Borel functions, namely the Baire class $\xi$ functions. Note that although in [7] it has been pointed out that there is a link between the two stratifications, from the point of view of reducibilities between sets of reals they clearly have a very different behaviour: in fact, we will prove that the Baire functions, contrarily to the case of the $D_{\xi}$ 's, induce a degree-structure which looks like the structure of

Date: November 20, 2018.

2000 Mathematics Subject Classification. 03E15, 03E60.

Key words and phrases. Determinacy, Wadge hierarchy.

Research supported by FWF (Austrian Research Fund) Grant P 19898-N18.

${ }^{1}$ As usual, we will always identify $\mathbb{R}$ with the Baire space ${ }^{\omega} \omega$ and call its elements "reals". 
the L-degrees. This result is obtained comparing again the Baire stratification with the Delta stratification, and showing that the first one gives reducibilities which are equivalent to (i.e. induce the same degree-structures as) the ones obtained glueing together chains of Borel-amenable sets of reductions. On the way of studying these classes of functions, we will introduce the notion of good Borel reducibility which considerably extends the definition of Borel-amenability given in [8] (in fact it includes, among others, all the examples quoted in this introduction): building on our previous results, we will give a new general method to study these reducibilities, which will lead to the following dichotomy theorem.

Theorem 1.1. Assume $\mathrm{AD}+\mathrm{DC}(\mathbb{R})$. If $\mathcal{F}$ is a good Borel set of reductions then it induces either a Lipschitz-like or a Wadge-like hierarchy of degrees.

This improves many of the results obtained in [8] and is a first step toward proving the naïve conjecture that the dichotomy above should hold for all "reasonable" Borel sets of reductions.

The paper is organized as follows: in Section 2 we will fix some notation and review some of the results about L-degrees and Borel-amenable reducibilities that will be needed for the rest of the work. (We will systematically omit the proofs of these results - the reader interested in some of them can consult [13], [12] or the more succinct [3] for Lipschitz degrees, and [8] for Borel-amenable sets of reductions.) In Section 3 we will give the definition of good Borel reducibility, while in Section 4 we will introduce the Strong Decomposition Property and prove some results which essentially form the framework of the proof of our dichotomy theorem. In Section 5 we will deal with the cases of Lipschitz functions and chains of reductions, and these results will in turn be used in Section 6 to analyze the hierarchies of degrees induced by Baire functions. Finally, in Section 7 we will show how to compare different degrees-structures (in particular showing how to obtain a certain hierarchy from the finer ones).

\section{Preliminaries}

Unless otherwise stated, we will always assume $\mathrm{ZF}+\mathrm{SLO}+\neg \mathrm{FS}+\mathrm{DC}(\mathbb{R})$ (see [8] and [3] for the definitions and for a brief account on these axioms). Anyway $\mathrm{SLO}^{\mathrm{L}}$ and $\neg \mathrm{FS}$ are easy consequences of $\mathrm{AD}$, thus one can also safely work in the most well-known theory ZF $+A D+D C(\mathbb{R})$. In both cases, all the "determinacy axioms" are used in a local way throughout the paper, thus e.g. to compare Borel sets is enough to assume Borel-determinacy. Our notation and terminology is quite standard, and we systematically refer the reader to [8] for the basic definitions. We just recall here that ${ }^{A} B$ denotes the set of all functions from $A$ into $B$, that a set $F \subseteq \omega_{2}$ is said flip-set whenever $\exists ! n(z(n) \neq w(n)) \Rightarrow(z \in F \Longleftrightarrow w \notin F)$ for every $z, w \in{ }^{\omega} 2$, and that, given any pointclass $\Gamma \subseteq \mathscr{P}(\mathbb{R})$, a function $f: \mathbb{R} \rightarrow \mathbb{R}$ is said $\Gamma$-function if $f^{-1}(D) \in \Gamma$ for every $D \in \Gamma$.

Let now $\mathcal{F} \subseteq \mathbb{R} \mathbb{R}$ be a family of functions which is closed under composition, contains $\mathrm{L}$ and admits a surjection $j: \mathbb{R} \rightarrow \mathcal{F}$, that is a so-called set of reductions. Recall that $A \leq_{\mathcal{F}} B \Longleftrightarrow A=f^{-1}(B)$ for some $f \in \mathcal{F}$ (notice that $A \leq_{\mathcal{F}} B \Longleftrightarrow$ $\neg A \leq_{\mathcal{F}} \neg B$ ), and let $<_{\mathcal{F}}$ be the strict relation associated to $\leq_{\mathcal{F}}$. Since $\leq_{\mathcal{F}}$ is a preorder, we can canonically define the equivalence relation $\equiv_{\mathcal{F}}$ and study the partial order $\leq$ induced by $\leq_{\mathcal{F}}$ on the equivalence classes of $\equiv_{\mathcal{F}}$, which are called $\mathcal{F}$ degrees. A set $A$ (or its $\mathcal{F}$-degree $[A]_{\mathcal{F}}$ ) is said to be $\mathcal{F}$-selfdual if and only if $A \leq_{\mathcal{F}}$ $\neg A$ (otherwise it is $\mathcal{F}$-nonselfdual), and $\left\{[A]_{\mathcal{F}},[\neg A]_{\mathcal{F}}\right\}$ is called nonselfdual pair 
whenever $A \not\rfloor_{\mathcal{F}} \neg A$. The Semi-Linear Ordering Principle for $\mathcal{F}$ is the statement

$$
\forall A, B \subseteq \mathbb{R}\left(A \leq_{\mathcal{F}} B \vee \neg B \leq_{\mathcal{F}} A\right) .
$$

Under $\mathrm{SLO}^{\mathcal{F}}$ we have that if $A$ and $B$ are $\leq_{\mathcal{F}}$-incomparable, then $B \equiv \equiv_{\mathcal{F}} \neg A$ : thus the ordering induced on the $\mathcal{F}$-degrees is almost a linear-order (it becomes indeed linear if each degree is identified with its dual). If now $\mathcal{F} \subseteq \mathcal{G} \subseteq \mathbb{R} \mathbb{R}$ are sets of reductions, then $\leq_{\mathcal{G}}$ is clearly coarser than $\leq_{\mathcal{F}}$ : hence $A \leq_{\mathcal{F}} B \Rightarrow A \leq_{\mathcal{G}} B$, if $A$ is $\mathcal{F}$-selfdual then it is also $\mathcal{G}$-selfdual, and $[A]_{\mathcal{F}} \subseteq[A]_{\mathcal{G}}$. Moreover the following basic lemma holds.

Lemma 2.1 (ZF). Let $\mathcal{F} \subseteq \mathcal{G} \subseteq \mathbb{R} \mathbb{R}$ be two sets of reductions. Then $\mathrm{SLO}^{\mathcal{F}} \Rightarrow \mathrm{SLO}^{\mathcal{G}}$, and assuming $\mathrm{SLO}^{\mathcal{F}}$ we have $\forall A, B \subseteq \mathbb{R}\left(A<_{\mathcal{G}} B \Rightarrow A<_{\mathcal{F}} B\right)$.

This lemma will be mostly used when $\mathcal{F}=\mathrm{L}$ : this in particular means that under our axiomatization we also have $\mathrm{SLO}^{\mathcal{F}}$ for every set of reductions $\mathcal{F}$. Moreover it easily implies that $\leq_{\mathcal{F}}$ is well-founded (since $\leq_{\mathrm{L}}$ is): therefore we can associate a rank $\|\cdot\|_{\mathcal{F}}$ to each set $A \subseteq \mathbb{R}$ (resp. $\mathcal{F}$-degree $[A]_{\mathcal{F}}$ ), and speak of successor and limit sets (resp. $\mathcal{F}$-degrees), and of the cofinality of a set (resp. of an $\mathcal{F}$-degree) with the obvious meaning. The next theorem sum up the general properties of sets of reductions - see Theorem 3.1 in [8]. Recall that given $A, B, A_{n} \in \mathbb{R}, \bigoplus_{n} A_{n}$ denotes the set $\bigcup_{n}\left(n^{\wedge} A_{n}\right)$, while $A \oplus B$ denotes $\bigoplus_{n} C_{n}$, where $C_{2 k}=A$ and $C_{2 k+1}=B$ for every $k \in \omega$.

Theorem 2.2. Let $\mathcal{F} \subseteq \mathbb{R} \mathbb{R}$ be a set of reductions. Then

i) $\operatorname{lh}\left(\leq_{\mathcal{F}}\right)=\Theta$, where $\Theta=\sup \{\alpha \mid f: \mathbb{R} \rightarrow \alpha$ for some surjection $f\}$;

ii) anti-chains have size at most 2 and are of the form $\left\{[A]_{\mathcal{F}},[\neg A]_{\mathcal{F}}\right\}$ for some set A;

iii) $\mathbb{R} \not \mathcal{F} \neg \mathbb{R}=\emptyset$ and if $A \neq \emptyset$, $\mathbb{R}$ then $\emptyset, \mathbb{R}<_{\mathcal{F}} A$;

iv) if $A \not \mathcal{F}_{\mathcal{F}} \neg A$ then $A \oplus \neg A$ is $\mathcal{F}$-selfdual and is the successor of both $A$ and $\neg A$. In particular, after an $\mathcal{F}$-nonselfdual pair there is a single $\mathcal{F}$-selfdual degree;

v) if $A_{0}<_{\mathcal{F}} A_{1}<_{\mathcal{F}} \ldots$ is a countable $\mathcal{F}$-chain of subsets of $\mathbb{R}$ then $\bigoplus_{n} A_{n}$ is $\mathcal{F}$-selfdual and is the supremum of these sets. In particular if $[A]_{\mathcal{F}}$ is limit of countable cofinality then $A \leq_{\mathcal{F}} \neg A$;

vi) if $A\rfloor_{\mathcal{F}} \neg A$ and $\mathcal{G} \subseteq \mathcal{F}$ is another set of reductions then $[A]_{\mathcal{F}}=[A]_{\mathcal{G}}$. In particular, $[A]_{\mathcal{F}}=[A]_{\mathrm{L}}$.

Thus to determine the hierarchy of degrees induced by some $\mathcal{F}$ we have only to understand what happens after a single selfdual degree and at limit levels of uncountable cofinality.

Given any set of reductions $\mathcal{F}$ (or even just any set of functions) we can define its characteristic set $\Delta_{\mathcal{F}}=\left\{A \subseteq \mathbb{R} \mid A \leq_{\mathcal{F}} \mathbf{N}_{\langle 0\rangle}\right\}$, which is formed by all sets $A \subseteq \mathbb{R}$ which are simple from the "point of view" of $\mathcal{F}$. As a simple excercise one can check that $\Delta_{\mathrm{D}_{\xi}}=\Delta_{\xi}^{0}$ for every countable $\xi$, and that $\Delta_{\text {Bor }}=\Delta_{1}^{1}$. It is easy to see that if $\mathcal{F}$ is closed under composition then every $f \in \mathcal{F}$ is a $\Delta_{\mathcal{F}}$-function (even if the converse is not always true - see [8] for a counter-example), thus it make sense to define the saturation of $\mathcal{F}$

$$
\operatorname{Sat}(\mathcal{F})=\left\{f \in{ }^{\mathbb{R}} \mathbb{R} \mid f \text { is a } \Delta_{\mathcal{F}} \text {-function }\right\},
$$

and to say that $\mathcal{F}$ is saturated just in case $\mathcal{F}=\operatorname{Sat}(\mathcal{F})$. Moreover it is easy to see that $\mathcal{F} \subseteq \mathcal{G}$ implies $\Delta_{\mathcal{F}} \subseteq \Delta_{\mathcal{G}}$ (the converse is not true in general, unless $\mathcal{G}=$ Bor or $\mathcal{G}=\mathrm{D}_{\xi}$ for some $\xi<\omega_{1}$, and $\mathbf{N}_{s} \in \Delta_{\mathcal{F}}$ for every $\left.s \in{ }^{<\omega} \omega\right)$. Finally, $\mathcal{F}$ is said to be Borel if $\left\{\mathbf{N}_{s} \mid s \in{ }^{<\omega} \omega\right\} \subseteq \Delta_{\mathcal{F}} \subseteq \Delta_{1}^{1}$, that is if $\mathcal{F} \subseteq$ Bor and $\mathcal{F}$ recognizes as simple all the basic clopen sets of $\mathbb{R}$.

Here are some basic facts about L-degrees: after a selfdual L-degree there is always another selfdual L-degree, and a limit L-degree is selfdual if and only if it is 
of countable cofinality, otherwise it is nonselfdual. Thus after a selfdual L-degree $[A]_{\mathrm{L}}$ there is always an $\omega_{1}$-chain of consecutive selfdual $\mathrm{L}$-degrees, and therefore the L-hierarchy looks like this:

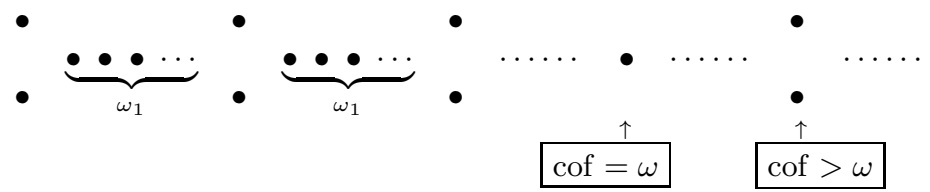

Any structure of this kind will be called Lipschitz-like.

Now we turn our attention to Borel-amenable sets of reductions.

Definition 1. A set of reductions $\mathcal{F}$ is Borel-amenable if:

i) $\operatorname{Lip} \subseteq \mathcal{F} \subseteq$ Bor;

ii) for every $\Delta_{\mathcal{F}}$-partition $\left\langle D_{n} \mid n \in \omega\right\rangle$ and every collection $\left\{f_{n} \mid n \in \omega\right\} \subseteq \mathcal{F}$ we have that

$$
f=\bigcup_{n \in \omega}\left(f_{n} \uparrow D_{n}\right) \in \mathcal{F}
$$

where Lip is the collection of all Lipschitz functions (with any constant).

As an example of Borel-amenable reducibility one can take any of the $D_{\xi}$ 's or Bor. It turns out that for every Borel-amenable set of reductions $\mathcal{F}$ there is some $\xi \leq \omega_{1}$ (called the level of $\mathcal{F}$ ) such that $\Delta_{\mathcal{F}}=\boldsymbol{\Delta}_{\xi}^{0}$ (where, with a little abuse of notation, we put $\left.\boldsymbol{\Delta}_{\omega_{1}}^{0}=\boldsymbol{\Delta}_{1}^{1}\right)$ : thus, in particular, $\operatorname{Sat}(\mathcal{F})$ is always either one of the $D_{\xi}$ 's or Bor. Moreover for any Borel-amenable set of reductions $\mathcal{F}$ we have the following lemma.

Lemma 2.3. Let $\left\langle D_{n} \mid n \in \omega\right\rangle$ be a $\Delta_{\mathcal{F}}$-partition of $\mathbb{R}$ and let $A \neq \mathbb{R}$.

a) $\forall n \in \omega\left(A \cap D_{n} \leq_{\mathcal{F}} A\right)$.

b) If $C \subseteq \mathbb{R}$ and $A \cap D_{n} \leq_{\mathcal{F}} C$ for every $n \in \omega$ then $A \leq_{\mathcal{F}} C$.

c) If $\forall n \in \omega\left(A \cap D_{n}<_{\mathcal{F}} A\right)$ then $A \leq_{\mathcal{F}} \neg A$. Moreover, if $D_{n}=\emptyset$ for all but finitely many n's then $A$ is not limit.

Let us say that $\mathcal{F}$ has the Decomposition Property (DP for short) if for every selfdual $A \notin \Delta_{\mathcal{F}}$ there is a $\Delta_{\mathcal{F}}$-partition $\left\langle D_{n} \mid n \in \omega\right\rangle$ of $\mathbb{R}$ such that $A \cap D_{n}<_{\mathcal{F}} A$ for every $n$ or, equivalently, if for every selfdual $A$ which is L-minimal in $[A]_{\mathcal{F}}$ one has that $A \leq_{\mathrm{L}} \neg A$ (and $A$ is either limit or successor of a nonselfdual pair with respect to $\leq_{\mathrm{L}}$ ). Using this property (which turns out to be a consequence of Borelamenability) we have proved in [8] that both after a single selfdual degree and at limit levels of uncountable cofinality there is a nonselfdual pair. Thus the hierarchy of degrees induced by $\mathcal{F}$ looks like this:

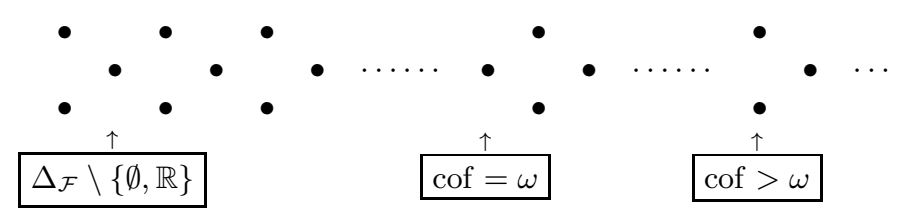

Any structure of this kind will be called Wadge-like. Notice that one gets both the Wadge hierarchy and the degree-structures induced by Bor and $D_{2}$ as particular instances of the previous result.

The DP allows also to compare different sets of reductions by means of the degree-structures induced by them. Let us say that two sets of reductions $\mathcal{F}$ and $\mathcal{G}$ are equivalent $(\mathcal{F} \simeq \mathcal{G}$ in symbols $)$ if they induce the same hierarchy of degrees, that is if for every $A, B \subseteq \mathbb{R}$ we have $A \leq_{\mathcal{F}} B \Longleftrightarrow A \leq_{\mathcal{G}} B$ : if $\mathcal{F}$ and $\mathcal{G}$ are Borel-amenable sets of reductions then

$$
\mathcal{F} \simeq \mathcal{G} \Longleftrightarrow \Delta_{\mathcal{F}}=\Delta_{\mathcal{G}},
$$


that is $\mathcal{F} \simeq \mathcal{G}$ if and only if their degree-structures coincide on the first nontrivial level. In particular, since $\Delta_{\mathcal{F}}=\Delta_{\text {Sat }(\mathcal{F})}$, we have that $\mathcal{F} \simeq \operatorname{Sat}(\mathcal{F})$.

We want to conclude this section by recalling how to construct, given an $\mathcal{F}$ selfdual set $A \subseteq \mathbb{R}$, where $\mathcal{F}$ is of level $\xi<\omega_{1}$, its successor degree(s). Fix an increasing sequence of ordinals $\left\langle\mu_{n} \mid n \in \omega\right\rangle$ cofinal in $\xi$, and a sequence of sets $P_{n}$ such that $P_{n} \in \boldsymbol{\Pi}_{\mu_{n}}^{0} \backslash \boldsymbol{\Sigma}_{\mu_{n}}^{0}$. Let $\langle\cdot, \cdot\rangle: \omega \times \omega \rightarrow \omega$ be any bijection, and for any zero-dimensional space ${ }^{2} \mathscr{X}$ define the homeomorphism

$$
\bigotimes^{\mathscr{X}}:{ }^{\omega}\left({ }^{\omega} \mathscr{X}\right) \rightarrow{ }^{\omega} \mathscr{X}:\left\langle x_{n} \mid n \in \omega\right\rangle \mapsto x=\bigotimes_{n}^{\mathscr{X}} x_{n},
$$

by letting $x(\langle n, m\rangle)=x_{n}(m)$, and, conversely, the "projections" $\pi_{n}^{\mathscr{X}}:{ }^{\omega} \mathscr{X} \rightarrow{ }^{\omega} \mathscr{X}$ by setting $\pi_{n}^{\mathscr{X}}(x)=\langle x(\langle n, m\rangle) \mid m \in \omega\rangle$ (clearly, every "projection" is surjective, continuous and open). Note that given a sequence of functions $f_{n}:{ }^{\omega} \mathscr{X} \rightarrow{ }^{\omega} \mathscr{X}$, we can use the homeomorphism $\otimes^{\mathscr{X}}$ to define the function

$$
\bigotimes^{\mathscr{X}}\left\langle f_{n} \mid n \in \omega\right\rangle=\bigotimes_{n}^{\mathscr{X}} f_{n}:{ }^{\omega} \mathscr{X} \rightarrow{ }^{\omega} \mathscr{X}: x \mapsto \bigotimes_{n}^{\mathscr{X}} f_{n}(x),
$$

and it is not hard to check that $\bigotimes_{n}^{\mathscr{X}} f_{n}$ is continuous if and only if all the $f_{n}$ 's are continuous. Now consider the sets

$$
\Sigma^{\xi}(A)=\left\{x \in \mathbb{R} \mid \exists n\left(\pi_{2 n}(x) \in P_{n} \wedge \forall i<n\left(\pi_{2 i}(x) \notin P_{i}\right) \wedge \pi_{2 n+1}(x) \in A\right)\right\}
$$

and

$$
\Pi^{\xi}(A)=\Sigma^{\xi}(A) \cup R_{\xi},
$$

where $R_{\xi}=\left\{x \in \mathbb{R} \mid \forall n\left(\pi_{2 n}(x) \notin P_{n}\right)\right\}$ : it turns out that for every $A \leq_{\mathcal{F}} \neg A$ the sets $\Sigma^{\xi}(A)$ and $\Pi^{\xi}(A)$ are $\leq \mathcal{F}_{\mathcal{F}}$-incomparable and are the immediate successors of $A$.

\section{Good Borel Reducibilities}

In [8] we have studied a special kind of Borel reducibilities but, as we will see later in this paper, there are also other "natural" sets of reductions which are not of this kind (namely Lipschitz functions, uniformly continuous functions, Baire functions, and so on). Thus our goal is to weaken the condition of Borel-amenability in order to be able to study also these other examples. Recall that the second condition of Borel-amenability says that $f=\bigcup_{n}\left(f_{n} \mid D_{n}\right) \in \mathcal{F}$ whenever $\left\{f_{n} \mid n \in \omega\right\} \subseteq \mathcal{F}$

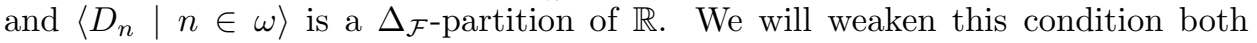
allowing to use only $f_{n}$ 's which are in $\mathrm{L}$ and using the concept of boundness (in a pointclass): a pointclass $\Lambda$ is ( $\mathrm{L}$-)bounded in an L-pointclass $\Gamma$ if there is some $A \in \Gamma$ such that $B \leq_{\mathrm{L}} A$ for every $B \in \Lambda$ (which in particular implies that $\Lambda \subseteq \Gamma$ ). Moreover, $\left\langle D_{n} \mid n \in \omega\right\rangle$ is a $\Gamma$-bounded partition of $\mathbb{R}$ if it is a $\Gamma$-partition of $\mathbb{R}$ such that $\left\{D_{n} \mid n \in \omega\right\}$ is bounded in $\Gamma$.

Definition 2. We say that $\mathcal{F}$ satisfies the partitioning condition (PC for short) if for every $\Delta_{\mathcal{F}}$-bounded partition $\left\langle D_{n} \mid n \in \omega\right\rangle$ of $\mathbb{R}$ and every collection $\left\{f_{n} \mid n \in\right.$ $\omega\} \subseteq \mathrm{L}$ one has that

$$
f=\bigcup_{n \in \omega}\left(f_{n} \uparrow D_{n}\right) \in \mathcal{F} .
$$

Notice that there are just three types of Borel reducibilities that can satisfy the PC:

TYPE I: $\Delta_{\mathcal{F}}=\left\{A \subseteq \mathbb{R} \mid A \leq_{\mathrm{L}} \mathbf{N}_{s}\right.$ for some $\left.s \in{ }^{<\omega} \omega\right\}$

TYPE II: $\Delta_{\mathcal{F}}=\Delta_{\xi}^{0}$ for some countable $\xi$ or $\Delta_{\mathcal{F}}=\Delta_{1}^{1}$;

TYPE III: $\Delta_{\mathcal{F}}=\Delta_{<\lambda}^{0}=\bigcup_{\mu<\lambda} \Delta_{\mu}^{0}$ for some countable limit ordinal $\lambda$.

\footnotetext{
${ }^{2}$ When $\mathscr{X}=\omega$ we will simply drop the symbol $\mathscr{X}$ in all the relevant notation.
} 
The proof of this fact is essentially the same of Proposition 4.3 in [8]. By Borel determinacy we need to consider just two cases $^{3}$, namely $\boldsymbol{\Delta}_{1}^{0} \subsetneq \Delta_{\mathcal{F}}$ and $\Delta_{\mathcal{F}} \subseteq \boldsymbol{\Delta}_{1}^{0}$. In the first case, assume that $\Delta_{\mathcal{F}} \neq \boldsymbol{\Delta}_{1}^{1}$ and that $\mathcal{F}$ is not of type III, and let $1<\xi<\omega_{1}$ be the smallest ordinal such that $\Delta_{\mathcal{F}} \subseteq \boldsymbol{\Delta}_{\xi}^{0}$. If $D \in \boldsymbol{\Delta}_{\xi}^{0}$, then there is some partition $\left\langle D_{n} \mid n \in \omega\right\rangle$ of $\mathbb{R}$ such that $D=\bigcup_{i \in I} D_{i}$ for some $I \subseteq \omega$ and $D_{n} \in \boldsymbol{\Pi}_{\mu_{n}}^{0}$ for some $\mu_{n}<\xi$ (see Theorem 4.2 in [7]). Since $\boldsymbol{\Delta}_{\mu}^{0} \subsetneq \Delta_{\mathcal{F}}$ for every $\mu<\xi$ (by minimality of $\xi$ ), we have that $\bigcup_{\mu<\xi} \Pi_{\mu}^{0} \subseteq \Delta_{\mathcal{F}}$ by Borel determinacy again, and hence that $\left\{D_{n} \mid n \in \omega\right\} \subseteq \bigcup_{\mu<\xi} \Pi_{\mu}^{0}$ is bounded in $\Delta_{\mathcal{F}}$ (when $\xi$ is limit use the fact that $\mathcal{F}$ is not of type III). Let $g_{0}, g_{1}$ be the constant functions with value $\overrightarrow{0}$ and $\overrightarrow{1}$, respectively, and put $f_{i}=g_{0}$ if $i \in I$ and $f_{i}=g_{1}$ otherwise. By the PC, $f=\bigcup_{n \in \omega}\left(f_{n}\left\lceil D_{n}\right) \in \mathcal{F}\right.$ and $f^{-1}\left(\mathbf{N}_{\langle 0\rangle}\right)=D$, i.e. $D \in \Delta_{\mathcal{F}}$ : therefore $\boldsymbol{\Delta}_{\xi}^{0} \subseteq \Delta_{\mathcal{F}}$ and $\mathcal{F}$ is of type II. Finally, the argument for the case $\Delta_{\mathcal{F}} \subseteq \boldsymbol{\Delta}_{1}^{0}$ is similar to the previous one (it suffices to prove that if $\mathcal{F}$ is not of type I then $\Delta_{\mathcal{F}}=\Delta_{1}^{0}$ ), and it is left to the reader.

As a corollary, one gets that $\Delta_{\mathcal{F}}$ is an algebra of sets (i.e. it is closed under complementation and finite intersections). Moreover, the $\mathbf{P C}$ implies $\mathcal{F} \supseteq \mathrm{L}$ : therefore, since $\Delta_{\mathcal{F}} \subseteq \Delta_{1}^{1}$ already implies that there is a surjection $j: \mathbb{R} \rightarrow \mathcal{F}$, a Borel set of functions $\mathcal{F}$ which satisfies the $\mathbf{P C}$ is also a set of reductions just in case it is closed under composition.

Another consequence of the $\mathbf{P C}$ is Lemma 4.4 of [8] (since any finite $\Delta_{\mathcal{F}}$-partition of $\mathbb{R}$ is obviously bounded in $\Delta_{\mathcal{F}}$ ): if $D \subseteq D^{\prime}$ are in $\Delta_{\mathcal{F}}$ and $A \subseteq \mathbb{R}$ is such that $A \cap D^{\prime} \neq \mathbb{R}$ then $A \cap D \leq_{\mathcal{F}} A \cap D^{\prime}$ (in particular, if $A \neq \mathbb{R}$ then $A \cap D \leq_{\mathcal{F}} A$ for every $D \in \Delta_{\mathcal{F}}$ ). Finally, the $\mathbf{P C}$ allows to reprove Lemma 2.3 (using almost the same argument) in case $\mathcal{F}$ is a Borel set of reductions which satisfies the $\mathbf{P C}$ (but non necessarily a Borel-amenable one) as soon as the partition $\left\langle D_{n} \mid n \in \omega\right\rangle$ is bounded in $\Delta_{\mathcal{F}}$ and part b) is replaced by the following condition:

$$
\text { if } C \subseteq \mathbb{R} \text { and } A \cap D_{n} \leq_{\mathrm{L}} C \text { for every } n \text { then } A \leq_{\mathcal{F}} C \text {. }
$$

Besides the $\mathbf{P C}$, there is also another condition which is somewhat hidden in the definition of Borel-amenability.

Definition 3. If $\Delta$ is an L-pointclass, we say that an arbitrary function $f: \mathbb{R} \rightarrow \mathbb{R}$ is $\sigma$-bounded (in $\Delta$ ) if for every countable collection $\left\{D_{n} \mid n \in \omega\right\}$ bounded in $\Delta$ one has that $\left\{f^{-1}\left(D_{n}\right) \mid n \in \omega\right\}$ is bounded in $\Delta$ as well (thus, in particular, $f$ is a $\Delta$-function). A set of functions $\mathcal{F}$ satisfies the $\sigma$-boundness condition ( $\sigma$-BC for short) if every $f \in \mathcal{F}$ is $\sigma$-bounded in $\Delta_{\mathcal{F}}$.

It is easy to check that if $\mathcal{F}$ is of type II then every countable collection $\left\{D_{n} \mid n \in\right.$ $\omega\} \subseteq \Delta_{\mathcal{F}}$ is bounded in $\Delta_{\mathcal{F}}$, thus $\sigma$-BC becomes relevant only when $\mathcal{F}$ is not of type II (this is the reason for which this condition was not explicity highlighted in [8]). On the other hand, if $\mathcal{F}$ is of type I or III the $\sigma$-BC turns out to be equivalent to the seemingly stronger statement "if $f \in \mathcal{F}$ and $\Gamma$ is bounded in $\Delta_{\mathcal{F}}$ (with $\Gamma$ of arbitrary size) then $\left\{f^{-1}(C) \mid C \in \Gamma\right\}$ is bounded in $\Delta_{\mathcal{F}}$ ": this is because in the cases under consideration $\Delta_{\mathcal{F}}$ has "countable cofinality" (i.e. there is a countable chain which is L-unbounded in $\Delta_{\mathcal{F}}$ ), and therefore from every pointclass L-unbounded in $\Delta_{\mathcal{F}}$ one can extract a countable subpointclass which is still L-unbounded in $\Delta_{\mathcal{F}}$.

We will see that the $\mathbf{P C}$ and the $\sigma$-BC are strong enough to civilize the hierarchy of degrees induced by $\mathcal{F}$, so let us give the following definition:

\footnotetext{
${ }^{3}$ The principle SLO ${ }^{\mathrm{L}}$ for Borel sets, which follows from Borel determinacy, implies that for every pair of L-pointclasses $\Gamma, \Lambda \subseteq \boldsymbol{\Delta}_{1}^{1}$ either $\Gamma \subseteq \Lambda$ or $\breve{\Lambda} \subseteq \Gamma$ : therefore if both $\Gamma$ and $\Lambda$ are selfdual either $\Gamma \subseteq \Lambda$ or $\Lambda \subseteq \Gamma$.
} 
Definition 4. A Borel set of reductions is said to be a good Borel reducibility if it satisfies both the $\mathbf{P C}$ and the $\sigma$-BC. The collection of all good Borel reducibilities will be denoted by GR.

Note that the Borel-amenable reducibilities form a proper subset of good Borel reducibilities of type II, as Lip $\nsubseteq \mathrm{D}_{\xi}^{L}$ for any $\xi<\omega_{1}$, where $\mathrm{D}_{\xi}^{\mathrm{L}}$ is the collection of those $f$ which are in $\mathrm{L}$ on a (countable) $\boldsymbol{\Delta}_{\xi}^{0}$-partition (in particular this proves that each $\mathrm{D}_{\xi}^{\mathrm{L}}$ does not contain any Borel-amenable set of reductions). In fact, one can easily check that the pseudoidentity $\mathrm{id}^{-}: \mathbb{R} \rightarrow \mathbb{R}: x \mapsto\langle x(n+1) \mid n \in \omega\rangle$ is such that for every countable partition $\left\langle D_{n} \mid n \in \omega\right\rangle$ and every family $\left\{f_{n} \mid n \in \omega\right\} \subseteq \mathrm{L}$ there is some $n_{0}$ such that $f_{n_{0}} \uparrow D_{n_{0}} \neq \mathrm{id}^{-} \uparrow D_{n_{0}}$ (the argument is based on the Baire Category Theorem and is almost identical to the one used in Remark 6.2 of $[8])$.

\section{The Strong Decomposition Property and the Dichotomy Theorem}

First we want to prove that every good Borel reducibility $\mathcal{F}$ has the following bounded version of the Decomposition Property.

Definition 5. A set $A \subseteq \mathbb{R}$ has the Strong Decomposition Property with respect to a Borel set of reductions $\mathcal{F}$ if there is a $\Delta_{\mathcal{F}}$-bounded partition $\left\langle D_{n} \mid n \in \omega\right\rangle$ of $\mathbb{R}$ such that $A \cap D_{n}<_{\mathcal{F}} A$ for every $n$.

A Borel set of reductions has the Strong Decomposition Property (SDP for short) if every $A \subseteq \mathbb{R}$ such that $A \leq_{\mathcal{F}} \neg A$ and $A \notin \Delta_{\mathcal{F}}$ has the Strong Decomposition Property with respect to $\mathcal{F}$.

Observe that if $\mathcal{F}$ is of type II then the SDP is equivalent to the DP by the observation following Definition 3.

Remark 4.1. If $\mathcal{F}$ satisfies the $\sigma$-BC, then $A$ has the Strong Decomposition Property with respect to $\mathcal{F}$ if and only if there is some $B$ in $[A]_{\mathcal{F}}$ which has the Strong Decomposition Property with respect to $\mathcal{F}$. In fact, let $f \in \mathcal{F}$ be a reduction of $A$ into $B$, and let $\left\langle D_{n}^{\prime} \mid n \in \omega\right\rangle$ be a $\Delta_{\mathcal{F}}$-bounded partition of $\mathbb{R}$ such that $B \cap D_{n}^{\prime}<_{\mathcal{F}} B$. Put $D_{n}=f^{-1}\left(D_{n}^{\prime}\right):\left\langle D_{n} \mid n \in \omega\right\rangle$ is a $\Delta_{\mathcal{F}}$-bounded partition of $\mathbb{R}$ by the $\sigma$-BC, and since $f$ witnesses $A \cap D_{n} \leq_{\mathcal{F}} B \cap D_{n}^{\prime}$ we have also $A \cap D_{n} \leq_{\mathcal{F}} B \cap D_{n}^{\prime}<_{\mathcal{F}} B \leq_{\mathcal{F}} A$.

If $\mathcal{F}$ is good (and satisfies a simple technical condition) then the SDP can also be recast in an equivalent way.

Proposition 4.2. Let $\mathcal{F} \in \mathrm{GR}$ be such that $k^{\frown} B \leq_{\mathcal{F}} B$ for every $k \in \omega$ and every $B \subseteq \mathbb{R}$ (we can require for instance that $\operatorname{Lip}(2) \subseteq \mathcal{F}$ ). Then for every $A \subseteq \mathbb{R}$ the following are equivalent:

i) A has the Strong Decomposition Property with respect to $\mathcal{F}$;

ii) if $B$ is $\mathrm{L}$-minimal in $[A]_{\mathcal{F}}$ then $B \leq_{\mathrm{L}} \neg B$.

Moreover, $B$ is either limit or successor of a nonselfdual pair with respect to $\leq_{\mathrm{L}}$.

Proof. If $A=\emptyset$ or $A=\mathbb{R}$ neither i) nor ii) can hold, thus we can assume $A \neq \emptyset, \mathbb{R}$. If $A$ has the Strong Decomposition Property with respect to $\mathcal{F}$, let $\left\langle D_{n} \mid n \in \omega\right\rangle$ be a $\Delta_{\mathcal{F}}$-bounded partition of $\mathbb{R}$ such that $A \cap D_{n}<_{\mathcal{F}} A$ for every $n$ and put $B_{n}=A \cap D_{n}$. Clearly we can not have that there is an $m \in \omega$ such that $B_{n} \leq_{\mathrm{L}} B_{m}$ for every $n \in \omega$, otherwise $A \leq_{\mathcal{F}} B_{m}$ by condition ( $\star$ ), a contradiction! Therefore $\forall m \exists n\left(B_{n} \mathbb{L}_{\mathrm{L}} B_{m}\right)$ and hence $B=\bigoplus_{n} B_{n}$ is L-selfdual. Moreover $B \leq_{\mathrm{L}} C$ for every $C \in[A]_{\mathcal{F}}$ since $B_{n}<_{\mathrm{L}} C$ for every $n \in \omega$ : on the other hand, $A \leq_{\mathcal{F}} B$ by condition $(\star)$ again, hence $B$ is L-minimal in $[A]_{\mathcal{F}}$.

Assume now that ii) holds. Recall that if $C$ is an arbitrary subset of $\mathbb{R}$ and $k \in \omega$, then $C_{\lfloor k\rfloor}$ denotes the set $\left\{x \in \mathbb{R} \mid k^{\frown} x \in C\right\}$. It is a classical fact 
that since $B$ is $\mathrm{L}$-selfdual we have $B_{\lfloor k\rfloor}<\mathrm{L} B$ for every $k \in \omega$, and hence by $\mathrm{L}$ minimality of $B$ in $[A]_{\mathcal{F}}$ we have also $B_{\lfloor k\rfloor}<_{\mathcal{F}} B$. Our technical condition implies that $B \cap \mathbf{N}_{\langle k\rangle} \leq_{\mathcal{F}} B_{\lfloor k\rfloor}$, and since $\left\langle\mathbf{N}_{\langle k\rangle} \mid k \in \omega\right\rangle$ is always bounded in $\Delta_{\mathcal{F}}$ then $B$ has the Strong Decomposition Property with respect to $\mathcal{F}$. But by Remark 4.1 this implies that $A$ has the Strong Decomposition Property with respect to $\mathcal{F}$ as well. The last part of the statement easily follows from our technical condition and the L-minimality of $B$ in $[A]_{\mathcal{F}}$, as if $B$ is the successor with respect to $\leq_{\mathrm{L}}$ of an $\mathrm{L}$-selfdual set $B^{\prime}$ then $B \equiv \mathrm{L} 0^{-} B^{\prime}$ (see e.g. [12] or [3] for a proof of this easy fact).

We will prove in Theorem 4.6 that the $\sigma$-BC already implies the SDP (also in absence of the $\mathbf{P C}$ and of the other technical condition), but first we need the next proposition, which is a deep application of the Martin-Monk method and a further strengthening of Theorem 16 in [4] and of Theorem 5.3 in [8]. As for the other results of this kind, we will use the following lemma (probably due to Kuratowski, see Lemma 5.1 in [8] and the references given there).

Lemma $4.3\left(\mathrm{ZF}+\mathrm{AC}_{\omega}(\mathbb{R})\right)$. Let $d$ be the usual metric on $\mathbb{R}, \tau$ the topology induced by $d$, and let $\xi$ be any nonzero countable ordinal. For any family $\left\{D_{n} \mid n \in \omega\right\} \subseteq \boldsymbol{\Delta}_{\xi}^{0}$ there is a metric $d^{\prime}$ on $\mathbb{R}$ such that

i) $\left(\mathbb{R}, \tau^{\prime}\right)$ is Polish and zero-dimensional, where $\tau^{\prime}$ is the topology induced by $d^{\prime}$;

ii) $\tau^{\prime}$ refines $\tau$;

iii) each $D_{n}$ is $\tau^{\prime}$-clopen;

iv) there is a countable clopen basis $\mathcal{B}^{\prime}$ for $\tau^{\prime}$ such that $\mathcal{B}^{\prime} \subseteq \boldsymbol{\Delta}_{\xi}^{0}$.

Proposition 4.4. Assume that $\mathcal{F}$ is of type I, II or III and has the $\sigma$-BC. Let $A \subseteq \mathbb{R}$ be such that $A \leq_{\mathcal{F}} \neg A, A \notin \Delta_{\mathcal{F}}$ and $A$ is L-minimal in its $\mathcal{F}$-degree. Then $A$ has the Strong Decomposition Property with respect to $\mathcal{F}$.

Proof. We start by considering the case in which $\mathcal{F}$ is of type III, the other cases will be treated in a similar way. First observe that since $A \leq_{\mathcal{F}} \neg A$ we have $A \not \mathbb{F}_{\mathcal{F}}$ $A \cap D \Longleftrightarrow A \cap D<_{\mathcal{F}} A$. Let $\xi<\omega_{1}$ be such that $\Delta_{\mathcal{F}}=\Delta_{<\xi}^{0}$, and let $f \in \mathcal{F}$ be any reduction of $A$ into $\neg A$. Toward a contradiction, assume that for every $\Delta_{\mathcal{F}}$ bounded partition $\left\langle D_{n} \mid n \in \omega\right\rangle$ of $\mathbb{R}$ there is some $n_{0} \in \omega$ such that $A \leq \mathcal{F} A \cap D_{n_{0}}$. We will construct three sequences ${ }^{4}$

$$
\left\langle C_{n} \mid n \in \omega\right\rangle,\left\langle d_{n} \mid n \in \omega\right\rangle,\left\langle f_{n} \mid n \in \omega\right\rangle
$$

such that for every $n \in \omega$ :

i) $C_{n} \in \Delta_{\mathcal{F}}$ and $A \leq_{\mathcal{F}} A \cap C_{n}$;

ii) $f_{n}: \mathbb{R} \rightarrow C_{n}$ is such that $f_{n}^{-1}\left(A \cap C_{n}\right)=A$ (i.e. $f_{n}$ reduces $A$ to $A \cap C_{n}$ ), and hence also $f_{n}^{-1}\left(\neg A \cap C_{n}\right)=\neg A$;

iii) $d_{n}$ is a metric on $\mathbb{R}$ such that the induced topology $\tau_{n}$ is zero-dimensional and Polish, refines all the previous $\tau_{m}$ 's $(m \leq n), C_{n}$ is $\tau_{n}$-clopen, and both $f_{n}:\left(\mathbb{R}, \tau_{n+1}\right) \rightarrow\left(\mathbb{R}, \tau_{n}\right)$ and $f_{n} \circ f:\left(\mathbb{R}, \tau_{n+1}\right) \rightarrow\left(\mathbb{R}, \tau_{n}\right)$ are continuous;

iv) for every $m \leq n$ and every $x, y \in C_{n+1}$

$$
d_{m}\left(g_{m} \circ \ldots \circ g_{n}(x), g_{m} \circ \ldots \circ g_{n}(y)\right)<2^{-n}
$$

where for each $i$ either $g_{i}=f_{i} \circ f\left\lceil C_{i+1}\right.$ or $g_{i}=f_{i}\left\lceil C_{i+1}\right.$.

Observe that by ii) we have that $f_{n} \circ f: \mathbb{R} \rightarrow C_{n}$ is such that

$$
\forall x \in C_{n+1}\left(x \in A \cap C_{n+1} \Longleftrightarrow f_{n} \circ f(x) \in \neg A \cap C_{n}\right),
$$

\footnotetext{
${ }^{4}$ The major difference from the present argument and the proof of Theorem 5.3 in [8] is that in this case we will not require that $C_{n+1} \subseteq C_{n}$, so that we will have to use some special $f_{n}$ 's (which "jump" from one $C_{n}$ into the next one) rather than the identity function.
} 
and that $f_{n}: \mathbb{R} \rightarrow C_{n}$ is such that

$$
\forall x \in C_{n+1}\left(x \in A \cap C_{n+1} \Longleftrightarrow f_{n}(x) \in A \cap C_{n}\right) .
$$

Having these sequences, we will be able to construct a flip-set (Wadge-reducible to $A$ ) using essentially the same argument contained in the proof of Theorem 16 in [4]. For every $z \in{ }^{\omega_{2}}$ put $g_{n}^{z}=f_{n} \circ f$ if $z(n)=1$, and $g_{n}^{z}=f_{n}$ otherwise. For every $n \in \omega$ choose some $y_{n+1} \in C_{n+1}$, and for every $m \leq n$ put $x_{n, m}^{z}=g_{m}^{z} \circ \ldots \circ g_{n}^{z}\left(y_{n+1}\right) \in C_{m}$. If we fix $m$ we get that $g_{m}^{z}\left(x_{n, m+1}^{z}\right)=x_{n, m}^{z}$ for every $n>m$, and that $\left\{x_{n, m}^{z} \mid n \geq m\right\} \subseteq C_{m}$ is a Cauchy sequence with respect to $d_{m}$ by (*). Therefore we can put $x_{m}^{z}=\lim _{n \rightarrow \infty} x_{n, m}^{z}$ and notice that $x_{m}^{z} \in C_{m}$ by the fact that $C_{m}$ is $\tau_{m}$-closed, and that $g_{m}^{z}\left(x_{m+1}^{z}\right)=x_{m}^{z}$ by continuity of $g_{m}^{z}$. Now it is easy to verify that $F=\left\{z \in \omega^{\omega} \mid x_{0}^{z} \in A\right\}$ is a flip-set.

The construction of the required sequences will be carried out by induction on $n$. To reach this goal we will construct also two auxiliary sequences

$$
\left\langle\mathcal{P}_{n} \mid n \in \omega\right\rangle,\left\langle\mu_{n} \mid n \in \omega\right\rangle
$$

such that:

1) $\mu_{n}$ is an increasing sequence of ordinals smaller than $\xi$ and $f_{n}$ is a $\boldsymbol{\Delta}_{\mu_{n}}^{0}$-function;

2) $\tau_{n}$ admits a countable basis $\mathcal{B}_{n} \subseteq \boldsymbol{\Delta}_{\mu_{n}}^{0}$;

3) $\mathcal{P}_{n}=\left\langle D_{m}^{n} \mid m \in \omega\right\rangle$ is a $\boldsymbol{\Delta}_{\mu_{n}}^{0}$-partition of $\mathbb{R}$ (in particular is bounded in $\Delta_{\mathcal{F}}$ ), $\mathcal{P}_{n+1}$ refines $\mathcal{P}_{n}, C_{n}=D_{m}^{n}$ for some $m$, and each $D_{m}^{n}$ is $\tau_{n}$-clopen.

At stage $n$ we will define $C_{n}, \mathcal{P}_{n}, f_{n}$ together with $d_{n+1}$ and $\mu_{n+1}$. First let $C_{0}=\mathbb{R}$, $\mathcal{P}_{0}$ be defined by $D_{0}^{0}=\mathbb{R}$ and $D_{m+1}^{0}=\emptyset, f_{0}=\mathrm{id}, \mu_{0}=1$, and $d_{0}$ be the usual metric on $\mathbb{R}$. By $\sigma$-BC there is some $\mu_{1}<\xi$ such that $\left\{f^{-1}\left(\mathbf{N}_{s}\right) \mid s \in{ }^{<\omega} \omega\right\} \subseteq \boldsymbol{\Delta}_{\mu_{1}}^{0}$, and we can let $d_{1}$ be the metric obtained applying Lemma 4.3 to this collection of sets (so that $f=f_{0} \circ f:\left(\mathbb{R}, \tau_{1}\right) \rightarrow\left(\mathbb{R}, \tau_{0}\right)$ is continuous). For the inductive step we need the following claim, which is analogous to Claim 5.3.1 of [8].

Claim 4.4.1. Let $D \subseteq \mathbb{R}$ be in $\Delta_{\mu}^{0}$ (for some $\mu<\xi$ ). If $A \leq_{\mathcal{F}} A \cap D$ then there is $g \in \mathrm{D}_{\mu}$ such that $g: \mathbb{R} \rightarrow D$ and $g$ reduces $A$ to $A \cap D$.

Proof of the Claim. We can assume $D \neq \emptyset, \mathbb{R}$ and $\neg A \cap D \neq \emptyset$, as if $D=\mathbb{R}$ then we can simply take $g$ to be the identity, while if $D=\emptyset$ or $D \subseteq A$ then $A \leq_{\mathcal{F}} A \cap D=D$ would contradict $A \notin \Delta_{\mathcal{F}}$. By the observation above we have that $A \not \mathbb{F}_{\mathcal{F}} A \cap D \Longleftrightarrow A \cap D<_{\mathcal{F}} A$, and by Lemma 2.1 and L-minimality of $A$ in its $\mathcal{F}$-degree we have that $A \cap D<_{\mathcal{F}} A \Longleftrightarrow A \cap D<_{\mathrm{L}} A$. Thus $A \leq_{\mathcal{F}} A \cap D$ implies that either $A \leq_{\mathrm{L}} A \cap D$ or, by SLO ${ }^{\mathrm{L}}, \neg A \leq_{\mathrm{L}} A \cap D$. If the second alternative holds, then since $A \cap D \leq \mathrm{D}_{\mu} A$ (see Lemma 4.4 in [8]) one also has $A \leq \mathrm{D}_{\mu} \neg A$ : thus in every case $A \leq \mathrm{D}_{\mu} A \cap D$. Let $g^{\prime} \in \mathrm{D}_{\mu}$ be a witness of this fact. Let $k \in \mathrm{D}_{\mu}$ be defined by $k(x)=x$ if $x \in D$ and $k(x)=y$ otherwise, where $y$ is any fixed point in $\neg A \cap D$. Letting $g=k \circ g^{\prime}$ it is easy to check that our claim holds. $\square$ Claim

Now suppose to have constructed all the sequences until stage $n$, that is $C_{i}, \mathcal{P}_{i}, f_{i}$, $d_{j}$ and $\mu_{j}$ for $i \leq n$ and $j \leq n+1$. Recall also from Claim 5.3 .2 of [8] that for every $m \leq n$ there is a $\Delta_{\mu_{m}}^{0}$-partition $\left\{C_{m}^{i} \mid i \in \omega\right\}$ of $\mathbb{R}$ such that $d_{m}$ - $\operatorname{diam}\left(C_{m}^{i}\right)<2^{-n}$ and $C_{m}^{i}$ is $\tau_{m}$-clopen for every $i \in \omega$. Fix $s \in{ }^{n+1} 2$ and let $g_{k}^{s}$ be defined (for every $k \leq n)$ by

$$
g_{k}^{s}= \begin{cases}f_{k} \circ f & \text { if } s(n)=1 \\ f_{k} & \text { if } s(n)=0\end{cases}
$$

Let $\left\langle D_{i, s}^{0} \mid i \in \omega\right\rangle$ be an enumeration of

$$
\left\{\left(g_{0}^{s} \circ \ldots \circ g_{n}^{s}\right)^{-1}\left(C_{0}^{i}\right) \cap D_{m}^{n} \mid i, m \in \omega\right\}
$$


and for $k<n$ let $\left\langle D_{i, s}^{k+1} \mid i \in \omega\right\rangle$ be an enumeration of

$$
\left\{\left(g_{k+1}^{s} \circ \ldots \circ g_{n}^{s}\right)^{-1}\left(C_{k+1}^{i}\right) \cap D_{j, s}^{k} \mid i, j \in \omega\right\} .
$$

Arguing by induction on $k \leq n$, it is not hard to see that $\left\langle D_{i, s}^{n} \mid i \in \omega\right\rangle$ is a $\boldsymbol{\Delta}_{\mu_{n+1}}^{0}{ }^{-}$ partition which refines $\mathcal{P}_{n}$, and that each $D_{i, s}^{n}$ is $\tau_{n+1}$-clopen since, by induction on $k<n$ again, one can prove that $g_{k}^{s} \circ \ldots \circ g_{n}^{s}:\left(\mathbb{R}, d_{n+1}\right) \rightarrow\left(\mathbb{R}, d_{k}\right)$ is continuous (and the $\tau_{n+1}$-clopen sets are contained in $\boldsymbol{\Delta}_{\mu_{n+1}}^{0}$, as $\mathcal{B}_{n+1} \subseteq \boldsymbol{\Delta}_{\mu_{n+1}}^{0}$ by inductive hypothesis).

Now fix an enumeration $\left\langle s_{l} \mid l<2^{n+1}\right\rangle$ of ${ }^{n+1} 2$ and inductively repeat the argument above but using $\left\langle D_{i, s_{l}}^{n} \mid i \in \omega\right\rangle$ instead of $\mathcal{P}_{n}$ at stage $l+1$. Let $\mathcal{P}_{n+1}=$ $\left\langle D_{m}^{n+1} \mid m \in \omega\right\rangle$ be the final partition of $\mathbb{R}$ obtained at stage $2^{n+1}$, and observe that one has again that $D_{m}^{n+1} \in \boldsymbol{\Delta}_{\mu_{n+1}}^{0}$ and that $D_{m}^{n+1}$ is $\tau_{n+1}$-clopen for every $m \in \omega$. Choose $\bar{m} \in \omega$ such that $A \leq_{\mathcal{F}} A \cap D_{\bar{m}}^{n+1}$ (such an $\bar{m}$ must exist by our assumption, since $\mathcal{P}_{n+1}$ is a $\Delta_{\mathcal{F}}$-bounded partition of $\mathbb{R}$ ), put $C_{n+1}=D_{\bar{m}}^{n+1}$, and let $f_{n+1}$ be the function obtained applying Claim 4.4.1 to $C_{n+1}$. We claim that there is some $\mu_{n+2} \geq \mu_{n+1}$ smaller than $\xi$ such that both

$$
\left\{f_{n+1}^{-1}(B) \mid B \in \mathcal{B}_{n+1}\right\} \subseteq \boldsymbol{\Delta}_{\mu_{n+2}}^{0} \quad \text { and } \quad\left\{\left(f_{n+1} \circ f\right)^{-1}(B) \mid B \in \mathcal{B}_{n+1}\right\} \subseteq \boldsymbol{\Delta}_{\mu_{n+2}}^{0} .
$$

In fact, the first part is obvious (since $f_{n+1}$ is a $\boldsymbol{\Delta}_{\mu_{n+1}}^{0}$-function and $\mathcal{B}_{n+1} \subseteq \boldsymbol{\Delta}_{\mu_{n+1}}^{0}$ ). For the second part, since $\left\{f_{n+1}^{-1}(B) \mid B \in \mathcal{B}_{n+1}\right\} \subseteq \boldsymbol{\Delta}_{\mu_{n+1}}^{0}$ is countable and bounded in $\Delta_{\mathcal{F}}$, by the $\sigma$-BC there must be some $\nu<\xi$ such that

$$
\left\{f^{-1}\left(f_{n+1}^{-1}(B)\right) \mid B \in \mathcal{B}_{n+1}\right\} \subseteq \boldsymbol{\Delta}_{\nu}^{0} .
$$

Put $\mu_{n+2}=\max \left\{\mu_{n+1}, \nu\right\}$ : it is easy to check that $\mu_{n+2}$ is as required.

Finally, apply Lemma 4.3 to the collection

$$
\left\{f_{n+1}^{-1}(B),\left(f_{n+1} \circ f\right)^{-1}(B) \mid B \in \mathcal{B}_{n+1}\right\} \subseteq \Delta_{\mu_{n+2}}^{0}
$$

to get $d_{n+2}$ with the desired properties (in particular, we have that both $f_{n+1} \circ$ $f:\left(\mathbb{R}, d_{n+1}\right) \rightarrow\left(\mathbb{R}, d_{n}\right)$ and $f_{n+1}:\left(\mathbb{R}, d_{n+1}\right) \rightarrow\left(\mathbb{R}, d_{n}\right)$ are continuous $)$. It is not hard to check that the sequences inductively constructed in this way satisfy all the conditions required, and this conclude the proof for the case when $\mathcal{F}$ is of type III.

Now let us consider the other possibilities for the set of reductions $\mathcal{F}$ : if $\mathcal{F}$ is of type I we can use the same argument as above but avoiding to construct the $\mu_{n}$ 's, letting $d_{n}$ be the usual metric on $\mathbb{R}$ for every $n \in \omega$ (thus dropping essentially condition iii), and constructing the partitions $\mathcal{P}_{n}$ in such a way that for each $n \in \omega$ there is some $k_{n}$ such that each element of $\mathcal{P}_{n}$, and in particular $C_{n}$, is L-reducible to $\mathbf{N}_{0^{\left(k_{n}\right)}}$ (the collection $\Delta_{k_{n}}=\left\{A \subseteq \mathbb{R} \mid A \leq_{\mathbf{L}} \mathbf{N}_{0^{\left(k_{n}\right)}}\right\}$ can be easily seen to be closed under finite intersections and unions $)^{5}$. Finally, if $\mathcal{F}$ is of type II one can repeat the argument above (in a slightly simpler way) taking advantage of the fact that every countable family of $\boldsymbol{\Delta}_{\xi}^{0}$ sets is bounded in $\boldsymbol{\Delta}_{\xi}^{0}$.

Remark 4.5. We can completely remove the hypothesis that $A$ is L-minimal in its $\mathcal{F}$-degree and reprove Proposition 4.4 assuming only $\mathrm{ZF}+\mathrm{AC}_{\omega}(\mathbb{R})+\neg \mathrm{FS}$ (thus giving essentially a direct proof of Theorem 4.6 under a weaker axiomatization) whenever $\mathcal{F}$ satisfies the following property (which is a consequence of $\mathbf{P C}$ ): if $D \in \Delta_{\mathcal{F}}$ and $f$ is a constant function then id $\uparrow D \cup f \uparrow \neg D$ is in $\mathcal{F}$. This is because in this case we can compose any reduction $f \in \mathcal{F}$ of $A$ into $A \cap D$ with the function $k$ defined in the proof of Claim 4.4.1 to get that if $A \leq_{\mathcal{F}} A \cap D$ then there is some $g \in \mathcal{F}$ such that $g: \mathbb{R} \rightarrow D$ and $g$ reduces $A$ to $A \cap D$. This fact can then be used to construct the $f_{n}$ 's and conclude the argument exactly in the same way. About the construction,

\footnotetext{
${ }^{5}$ One has also to modify the statement of Claim 4.4 .1 in the following way: "Let $D \subseteq \mathbb{R}$ be in $\Delta_{k_{n}}$ (for some $k_{n} \in \omega$ ). If $A \leq_{\mathcal{F}} A \cap D$ then there is a $g: \mathbb{R} \rightarrow D$ such that $g$ is a $\Delta_{k_{n}}$-function which reduces $A$ to $A \cap D$ ".
} 
one should just be careful in the inductive step, and check that an ordinal $\mu_{n+2}$ with the desired properties exists because both $f_{n+1}$ and $f$ are $\sigma$-bounded in $\Delta_{\mathcal{F}}$, and the composition of $\sigma$-bounded functions is still $\sigma$-bounded.

Now we are ready to prove the Strong Decomposition Theorem.

Theorem 4.6. Let $\mathcal{F}$ be a Borel set of reductions which satisfies the $\sigma-\mathbf{B C}$. Then $\mathcal{F}$ has the SDP.

Proof. Assume first that $\mathcal{F}$ is of type I, II or III. Let $A \leq_{\mathcal{F}} \neg A \notin \Delta_{\mathcal{F}}$ and let $B$ be L-minimal in $[A]_{\mathcal{F}}$ : then $B$ has the Strong Decomposition Property with respect to $\mathcal{F}$ by Proposition 4.4, which by Remark 4.1 implies that $A$ has the Strong Decomposition Property with respect to $\mathcal{F}$ as well.

Now assume that $\mathcal{F}$ is not of type I-III, i.e. that $\boldsymbol{\Delta}_{<\xi}^{0} \subsetneq \Delta_{\mathcal{F}} \subsetneq \boldsymbol{\Delta}_{\xi}^{0}$ for some countable $\xi$ (notice that in this case we will not use the $\sigma$-BC). By Proposition 3.3 of [8] we have that $\mathcal{F} \subseteq \mathrm{D}_{\xi}$, thus if $A \leq \mathcal{F} \neg A$ we have also $A \leq_{\mathrm{D}_{\xi}} \neg A$. By the SDP for $\mathrm{D}_{\xi}$, there must be a $\boldsymbol{\Delta}_{\xi}^{0}$-partition $\left\langle D_{n}^{\prime} \mid n \in \omega\right\rangle$ of $\mathbb{R}$ such that $A \cap D_{n}^{\prime}<_{\mathrm{D}_{\xi}} A$ for every $n$. This partition can be refined to a $\bigcup_{\mu<\xi} \Pi_{\mu}^{0}$-partition $\left\langle D_{n} \mid n \in \omega\right\rangle$ of $\mathbb{R}$ with the same property, that is such that $A \cap D_{n}<_{\mathrm{D}_{\xi}} A$ for every $n$. But $\bigcup_{\mu<\xi} \Pi_{\mu}^{0}$ is easily seen to be bounded in $\Delta_{\mathcal{F}}$, and $A \cap D_{n}<_{\mathcal{F}} A$ by $\mathrm{SLO}^{\mathcal{F}}$.

The Strong Decomposition Theorem (together with part $c$ ) of Lemma 2.3) implies that $A \leq{ }_{\mathcal{F}} \neg A$ if and only if $A$ has the Strong Decomposition Property with respect to $\mathcal{F}$, thus if $\mathcal{F}$ is good we can adjoin the condition $A \leq_{\mathcal{F}} \neg A$ to the equivalents of Proposition 4.2. Moreover, as a corollary of Theorem 4.6 one gets also that if $\mathcal{F}$ is a good Borel reducibility then at limit levels of uncountable cofinality there is a nonselfdual pair.

Corollary 4.7. Let $\mathcal{F}$ be a good Borel set of reductions and let $[A]_{\mathcal{F}}$ be a selfdual limit degree. Then $[A]_{\mathcal{F}}$ is of countable cofinality.

Proof. Let $\left\langle D_{n} \mid n \in \omega\right\rangle$ be a $\Delta_{\mathcal{F}}$-bounded partition of $\mathbb{R}$ such that $A \cap D_{n}<_{\mathcal{F}} A$ and

$$
\forall n \in \omega \exists m \in \omega\left(A \cap D_{n}<_{\mathcal{F}} A \cap D_{m}\right)
$$

(such a partition must exist by Theorem 4.6 and by the fact that $[A]_{\mathcal{F}}$ is limit): then $\mathcal{A}=\left\{\left[A \cap D_{n}\right]_{\mathcal{F}} \mid n \in \omega\right\}$ witnesses that $[A]_{\mathcal{F}}$ is of countable cofinality (use condition $(\star)$ and the fact that if $A \cap D_{n} \leq_{\mathcal{F}} B$ for every $n$ then $A \cap D_{n}<_{\mathrm{L}} B$ by $(\dagger)$ and Lemma 2.1).

The Strong Decomposition Theorem implies also that we can compare good Borel reducibilities with respect to the degree-structures induced by them.

Theorem 4.8. Let $\mathcal{F}$ and $\mathcal{G}$ be two Borel sets of reductions such that $\mathcal{G}$ has the SDP , $\mathcal{F}$ satisfies the $\mathbf{P C}$, and $\Delta_{\mathcal{G}} \subseteq \Delta_{\mathcal{F}}$. Then for every $A, B \subseteq \mathbb{R}$

$$
A \leq_{\mathcal{G}} B \Rightarrow A \leq_{\mathcal{F}} B .
$$

In particular, if $\mathcal{F}$ and $\mathcal{G}$ are good Borel reducibilities then $\mathcal{F} \simeq \mathcal{G}$ if and only if $\Delta_{\mathcal{F}}=\Delta_{\mathcal{G}}$.

The proof is identical to the one of Theorem 4.7 in [8] — the only obvious modification is that we have to use SDP instead of DP. Using Theorem 4.8, one can now obtain the dichotomy theorem for good Borel reducibilities (which is simply a more detailed recasting of Theorem 1.1).

Theorem 4.9. Let $\mathcal{F}$ be a good Borel reducibility. Then one of the following holds: 
i) $\mathcal{F}$ induces a Wadge-like degree-structure:

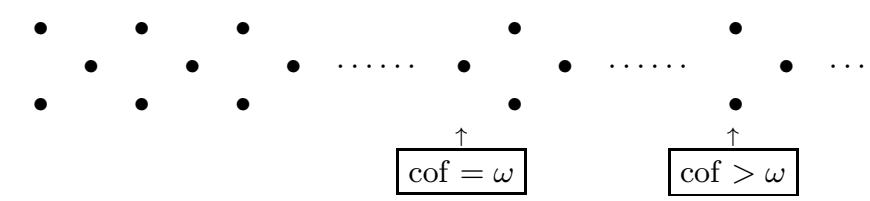

ii) $\mathcal{F}$ induces a Lipschitz-like degree-structure:

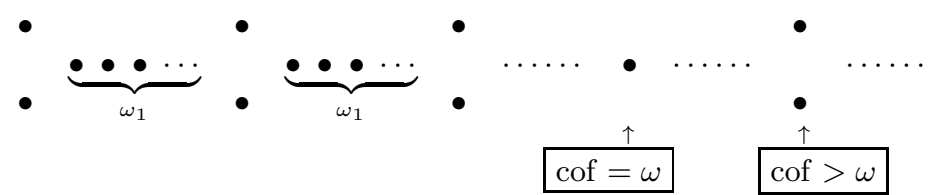

In particular, the first alternative holds if $\mathcal{F}$ is of type II, while the second alternative holds if $\mathcal{F}$ is either of type I or of type III.

The proof of this theorem can be obtained by choosing some "canonical" representative for each equivalence class induced by the equivalence relation $\simeq$ on $\mathrm{GR}$, and by studying the degree-structure induced by it. These examples are, respectively: Lip for the collection of the good Borel reducibilities of type I, $\mathrm{D}_{\xi}$ or Bor for the $\mathcal{F}$ 's of type II such that $\Delta_{\mathcal{F}}=\boldsymbol{\Delta}_{\xi}^{0}$ (for each $\xi \leq \omega_{1}$ ), and the chain of reductions $\bigcup_{\mu<\xi} \mathrm{D}_{\mu}$ for the $\mathcal{F}$ 's of type III such that $\Delta_{\mathcal{F}}=\Delta_{<\xi}^{0}$ (for every countable limit $\xi$ ). The degree-structures of Bor and $\mathrm{D}_{\xi}$ have already been determined in [8], while the degree-structures of Lip and of the chains of reductions will be determined in the next section of this paper (one can check that all these results are coherent with the description given in Theorem 4.9). Therefore it will be enough to apply Theorem 4.8 , with $\mathcal{G}$ being the suitable "canonical" representative (i.e. the canonical example such that $\Delta_{\mathcal{F}}=\Delta_{\mathcal{G}}$ ), to get the result for an arbitrary good Borel reducibility $\mathcal{F}$.

\section{Good Borel Reducibilities of TyPe I AND III}

In this section we will analyze the degree-structures induced by Lip and by (regular) chains of reductions, showing in particular that they are all Lipschitz-like. This will complete the proof of Theorem 4.9.

5.1. Lipschitz functions. First we want to prove that Lip is a good Borel reducibility of type I, and this practically amounts to compute that

$$
\Delta_{\text {Lip }}=\bigcup_{0 \neq n \in \omega}\left[\mathbf{N}_{0^{(n)}}\right]_{\mathrm{L}} \cup\{\emptyset, \mathbb{R}\}=\bigcup_{s \in<\omega \omega}\left[\mathbf{N}_{s}\right]_{\llcorner} \cup\{\emptyset\} .
$$

One direction is obvious, so we will just prove $\Delta_{\text {Lip }} \subseteq \bigcup_{0 \neq n \in \omega}\left[\mathbf{N}_{0^{(n)}}\right]_{\mathrm{L}} \cup\{\emptyset, \mathbb{R}\}$. Let $\emptyset \neq A \in \Delta_{\text {Lip }}$ : by definition there are $f \in \operatorname{Lip}$ and $n \in \omega$ such that $f \in \operatorname{Lip}\left(2^{n}\right)$ and $f^{-1}\left(\mathbf{N}_{\langle 0\rangle}\right)=A$. We want to show that $S=\left\{s \in{ }^{n+1} \omega \mid f\left(\mathbf{N}_{s}\right) \subseteq \mathbf{N}_{\langle 0\rangle}\right\}$ is such that $A=\bigcup_{s \in S} \mathbf{N}_{s}$ : since the set on the right of the equation is clearly L-reducible to $\mathbf{N}_{0^{(n+1)}}$, this will finish the proof. Clearly $\bigcup_{s \in S} \mathbf{N}_{s} \subseteq A$. For the other direction, pick any $x \in A$ : being $f$ a reduction of $A$ into $\mathbf{N}_{\langle 0\rangle}, f(x) \in \mathbf{N}_{\langle 0\rangle}$. Since $f \in \operatorname{Lip}\left(2^{n}\right)$, $d(f(x), f(y)) \leq 2^{-1}$ for every $y \in \mathbf{N}_{x \uparrow(n+1)}$, which means $f\left(\mathbf{N}_{x \uparrow(n+1)}\right) \subseteq \mathbf{N}_{\langle 0\rangle}$ : but then $x\left\lceil(n+1) \in S\right.$ and hence $x \in \bigcup_{s \in S} \mathbf{N}_{s}$.

Since we have just proved that Lip $\in$ GR, to determine the degree-structure induced by Lip we have only to understand what happens after a selfdual degree. Given any set $A \subseteq \mathbb{R}$ define

$$
s_{\text {Lip }}(A)=\bigoplus_{n} 0^{(n) \frown} A .
$$

We want to prove that if $A \leq_{\text {Lip }} \neg A$ then $\left[s_{\text {Lip }}(A)\right]_{\text {Lip }}$ is selfdual and is the immediate successor of $[A]_{\text {Lip. }}$. This will prove that after a selfdual Lip-degree there is 
always another selfdual Lip-degree, and that Lip induce a degree-structure which is Lipschitz-like.

Proposition 5.1. Let $A \subseteq \mathbb{R}$ be Lip-selfdual. Then $s_{\text {Lip }}(A) \leq_{\text {Lip }} \neg s_{\text {Lip }}(A), A<_{\text {Lip }}$ $s_{\text {Lip }}(A)$ and there is no $B$ such that $A<$ Lip $B<$ Lip $s_{\text {Lip }}(A)$.

Proof. Let $A$ be L-minimal in its Lip-degree and observe that one has $A \leq_{\mathrm{L}} \neg A$ by Proposition 4.2 (note that obviously $\operatorname{Lip}(2) \subseteq \mathrm{Lip}$ ). This implies that $A<\mathrm{L} 0^{\frown} A<\mathrm{L}$ $\ldots<_{\mathrm{L}} 0^{(n)}-A<_{\mathrm{L}} \ldots$, and hence that $s_{\mathrm{Lip}}(A) \leq_{\mathrm{L}} \neg s_{\mathrm{Lip}}(A)$. Moreover it is clear that for every $n \in \omega$ we have $A \leq_{\mathrm{L}} 0^{(n)} \wedge A$ and that $0^{(n)} \frown A \leq_{\mathrm{Lip}} A$ via a function $f \in \operatorname{Lip}\left(2^{n}\right)$. If $B<_{\text {Lip }} s_{\text {Lip }}(A)$ we have that $B<_{\mathrm{L}} s_{\text {Lip }}(A)$ by Lemma 2.1, which in turn implies $B \leq_{\mathrm{L}} 0^{(n)}-A$ for some $n \in \omega$ : hence $B \leq_{\mathrm{Lip}} A$. Therefore it remains only to prove that $s_{\text {Lip }}(A) \mathbb{L}_{\text {Lip }} A$. Toward a contradiction, assume that there is $f \in$ Lip such that $f^{-1}(A)=s_{\text {Lip }}(A)$, and let $n$ be the smallest natural number such that $f \in \operatorname{Lip}\left(2^{n}\right)$, so that $f\left(\mathbf{N}_{0^{(n+1)}}\right) \subseteq \mathbf{N}_{\langle k\rangle}$ for some $k \in \omega$. Let $g$ be defined by $g(x)=f(x)$ if $x \in \mathbf{N}_{0^{(n+1)}}$ and $g(x)=(k+1) \frown \overrightarrow{0}$ otherwise: then $g \in \operatorname{Lip}\left(2^{n+1}\right)$ and reduces $0^{(n+1)}-A$ to $A \cap \mathbf{N}_{\langle k\rangle}$. But it is easy to check that $A \cap \mathbf{N}_{\langle k\rangle} \leq_{\text {Lip }} A_{\lfloor k\rfloor}$ and $A_{\lfloor k\rfloor}<\mathrm{L} A$ : therefore, by L-minimality of $A$ in its Lip-degree we would have that

$$
0^{(n+1) \frown A \leq \text { Lip }} A_{\lfloor k\rfloor}<\text { Lip } A,
$$

a contradiction!

The definition of the successor operator $s_{\text {Lip }}$, allows also to obtain a way to construct the Lip-degrees from the L-degrees. In fact, if $[A]_{\text {Lip }}$ is nonselfdual, then $[A]_{\text {Lip }}=[A]_{\mathrm{L}}$ by Theorem 2.2, while if $A \leq_{\mathrm{L}} \neg A$ and $A$ is L-minimal in its Lipdegree, then $[A]_{\text {Lip }}$ is exactly $\bigcup_{n \in \omega}\left[0^{(n)}-A\right]_{\mathrm{L}}$.

As an application of Theorem 4.8, let us now consider the set of the uniformly continuous functions (which will be denoted by UCont): it turns out (perhaps rather surprisingly, since uniform continuity is just a weak "refinement" of continuity) that UCont is equivalent to Lip (rather than to W), and thus gives a hierarchy of degrees which is Lipschitz-like. In fact, one can easily check that UCont is a good Borel reducibility and that $\Delta_{U \text { Cont }}=\bigcup_{s \in<\omega_{\omega}}\left[\mathbf{N}_{s}\right]_{\mathrm{L}} \cup\{\emptyset\}: \mathbf{N}_{s}$ is reducible to $\mathbf{N}_{\langle 0\rangle}$ via a function in $\operatorname{Lip}\left(2^{\operatorname{lh}(s)}\right) \subseteq$ UCont, while if $f$ is uniformly continuous then there must be some $m \in \omega$ such that for every $x, y \in \mathbb{R}$

$$
d(x, y) \leq 2^{-m} \Rightarrow d(f(x), f(y)) \leq 2^{-1},
$$

and thus, in particular, $f$ can not reduce $\bigoplus_{n} \mathbf{N}_{0(n)}$ to $\mathbf{N}_{\langle 0\rangle}$ (the argument is similar to the one used in Proposition 5.1). This proves also that $\Delta_{\text {Lip }}=\Delta_{U \text { Cont }}$, and that UCont is of type I: therefore Lip $\simeq$ UCont by Theorem 4.8. Moreover it is not hard to check that UCont is maximal among the good Borel reducibilities of type I, since the fact that $\mathcal{F}$ is of type I and satisfies the $\sigma$-BC implies $\mathcal{F} \subseteq$ UCont - UCont is exactly the collection of all $\sigma$-bounded $\Delta_{\text {Lip }}$-functions.

5.2. Chains of reductions. A (countable) chain of (Borel-amenable sets of) reductions is simply any sequence $\overrightarrow{\mathcal{F}}=\left\langle\mathcal{F}_{n} \mid n \in \omega\right\rangle$ of Borel-amenable sets of reductions. To each chain of reductions we will associate the unique sequence of ordinals $\left\langle\mu_{n} \mid n \in \omega\right\rangle$ such that $1 \leq \mu_{n} \leq \omega_{1}$ and $\Delta_{\mathcal{F}_{n}}=\Delta_{\mu_{n}}^{0}$ for every $n \in \omega$, which will be called the type of $\overrightarrow{\mathcal{F}}$. Moreover we will say that $\overrightarrow{\mathcal{F}}$ is of rank $\omega_{1}$ if $\mu_{n}=\omega_{1}$ for some $n \in \omega$, and of rank $1 \leq \xi<\omega_{1}$ if $\mu_{n}<\omega_{1}$ for every $n \in \omega$ and $\xi=\sup \left\{\mu_{n}+1 \mid n \in \omega\right\}$. A chain of reductions will be called regular if each $\mathcal{F}_{n}$ is saturated and $\mathcal{F}_{n} \subsetneq \mathcal{F}_{n+1}$ for every $n$ (in particular, the rank $\xi$ of $\overrightarrow{\mathcal{F}}$ must be 
countable and limit ${ }^{6}$. Note that in this case $\bigcup_{n} \mathcal{F}_{n}=\bigcup_{\mu<\xi} \mathrm{D}_{\mu}$ is a Borel set of reductions, and since

$$
\Delta_{\bigcup_{n} \mathcal{F}_{n}}=\bigcup_{n} \Delta_{\mathcal{F}_{n}}=\bigcup_{n} \Delta_{\mu_{n}}^{0}=\Delta_{<\xi}^{0}
$$

one can check that $\bigcup_{n} \mathcal{F}_{n}$ is good and of type III: thus, as we have already pointed out, regular chains of reductions provide a canonical way to construct good Borel reducibilities of type III (one for each possible characteristic set). From now onward, we will fix some limit $\xi<\omega_{1}$ and consider a regular chain of reductions $\overrightarrow{\mathcal{F}}=$ $\left\langle\mathcal{F}_{n} \mid n \in \omega\right\rangle$ of rank $\xi$. By Corollary 4.7, in order to describe the structure of degrees induced by ${ }^{7} \leq_{\overrightarrow{\mathcal{F}}}$ we have only to determine what happens after a selfdual degree: this can be done using the following proposition about Borel-amenable sets of reductions.

Proposition 5.2. Let $\mathcal{G}$ and $\mathcal{G}^{\prime}$ be two Borel-amenable sets of reductions such that $\Delta_{\mathcal{G}} \subsetneq \Delta_{\mathcal{G}^{\prime}}$ (i.e. such that $\mathcal{G}$ is of level strictly smaller than $\mathcal{G}^{\prime}$ ). Let $A \leq_{\mathcal{G}} \neg A$ and $B$ be a (nonselfdual) successor of $A$ with respect to $\leq_{\mathcal{G}}$ : then $B \leq_{\mathcal{G}^{\prime}} A$. In particular, if $\mu$ is the level of $\mathcal{G}$ and $A \leq_{\mathcal{G}} \neg A$, then $\Sigma^{\mu}(A) \leq_{\mathcal{G}^{\prime}} A$ and $\Pi^{\mu}(A) \leq_{\mathcal{G}^{\prime}} A$.

Proof. If $\mathcal{G}$ and $B$ are as above then either $B \equiv_{\mathcal{G}} \Sigma^{\mu}(A)$ or $B \equiv_{\mathcal{G}} \Pi^{\mu}(A) \equiv_{\mathcal{G}}$ $\neg \Sigma^{\mu}(A)$, and since $\Delta_{\mathcal{G}} \subseteq \Delta_{\mathcal{G}^{\prime}}$ implies $A \leq_{\mathcal{G}} B \Rightarrow A \leq_{\mathcal{G}^{\prime}} B$ for every $A, B \subseteq \mathbb{R}$ (by Theorem 4.8), it is enough to prove $\Sigma^{\mu}(A) \leq_{\mathcal{G}^{\prime}} A$. Let $P_{n}$ and $R_{\mu}$ be the sets used to define the operation $\Sigma^{\mu}$, and define $F_{0}=\left\{x \in \mathbb{R} \mid \pi_{0}(x) \in P_{0}\right\}$ and $F_{n+1}=\left\{x \in \mathbb{R} \mid \pi_{2(n+1)}(x) \in P_{n+1} \wedge \forall i \leq n\left(\pi_{2 i}(x) \notin P_{i}\right)\right\}$. Clearly, every $F_{n} \in \boldsymbol{\Delta}_{\mu}^{0} \subseteq \Delta_{\mathcal{G}^{\prime}}$, and since $R_{\mu} \in \boldsymbol{\Pi}_{\mu}^{0}$ and $\boldsymbol{\Delta}_{\mu}^{0} \subsetneq \Delta_{\mathcal{G}^{\prime}}$, we have also $R_{\mu} \in \Delta_{\mathcal{G}^{\prime}}$ (as $\boldsymbol{\Sigma}_{\mu}^{0} \cup \boldsymbol{\Pi}_{\mu}^{0} \subseteq \Delta_{\mathcal{G}^{\prime}}$ by Borel-determinacy). On each of these sets we can continuously reduce $\Sigma^{\mu}(A)$ to $A$ using $\pi_{2 n+1}$ on the $F_{n}$ 's and a constant function with value $\bar{y} \notin A$ on $R_{\mu}\left(A \neq \mathbb{R}\right.$ as $\left.A \leq_{\mathcal{G}} \neg A\right)$, hence $\Sigma^{\mu}(A) \leq_{\mathrm{D}_{\mu^{\prime}}} A$, where $\mu^{\prime}$ is the level of $\mathcal{G}^{\prime}$. But since $\mathcal{G}^{\prime} \simeq \mathrm{D}_{\mu^{\prime}}^{\mathrm{W}}$ we are done.

Theorem 5.3. If $A \leq_{\overrightarrow{\mathcal{F}}} \neg A$ then there is some $B \leq_{\overrightarrow{\mathcal{F}}} \neg B$ with the property that $A<_{\overrightarrow{\mathcal{F}}} B$ and there is no $C$ such that $A<_{\overrightarrow{\mathcal{F}}} C<_{\overrightarrow{\mathcal{F}}} B$. Thus after an $\overrightarrow{\mathcal{F}}$-selfdual degree there is another $\overrightarrow{\mathcal{F}}$-selfdual degree.

Proof. Taking $A$ to be L-minimal in $[A]_{\overrightarrow{\mathcal{F}}}$, by Proposition 4.2 and the fact that $\overrightarrow{\mathcal{F}}$ has the SDP we can assume $A \leq_{\mathrm{L}} \neg A$ (hence, in particular, $A \leq_{\mathcal{F}_{n}} \neg A$ for every $n \in \omega)$. Let $\left\langle\mu_{n} \mid n \in \omega\right\rangle$ be the type of $\overrightarrow{\mathcal{F}}$ and define the successor operator $s_{\overrightarrow{\mathcal{F}}}$ by letting

$$
B=s_{\overrightarrow{\mathcal{F}}}(A)=\bigoplus_{n} \Sigma^{\mu_{n}}(A) .
$$

Clearly $A \leq_{\mathrm{L}} s_{\overrightarrow{\mathcal{F}}}(A)$, and if $C<_{\overrightarrow{\mathcal{F}}} s_{\overrightarrow{\mathcal{F}}}(A)$ then we have also $C<_{\mathrm{L}} s_{\overrightarrow{\mathcal{F}}}(A)$, which implies $C \leq_{\mathrm{L}} \Sigma^{\mu_{n}}(A)$ for some $n \in \omega$ : but since $\Sigma^{\mu_{n}}(A) \leq_{\mathcal{F}_{n+1}} A$ by Proposition 5.2 , we have also $C \leq_{\overrightarrow{\mathcal{F}}} A$. Finally, the fact that $s_{\overrightarrow{\mathcal{F}}}(A) \leq_{\overrightarrow{\mathcal{F}}} \neg s_{\overrightarrow{\mathcal{F}}}(A)$ will follow from the fact that $\Sigma^{\mu_{n}}(A)<_{\mathrm{L}} \Sigma^{\mu_{n+1}}(A)$ for every $n \in \omega$ (since this implies $s_{\overrightarrow{\mathcal{F}}}(A) \leq_{\mathrm{L}}$ $\left.\neg s_{\overrightarrow{\mathcal{F}}}(A)\right)$. To see this, recall that $\Sigma^{\mu_{n}}(A) \leq_{\mathcal{F}_{n+1}} A$ while $A<_{\mathcal{F}_{n+1}} \Sigma^{\mu_{n+1}}(A)$, which implies $\Sigma^{\mu_{n}}(A)<_{\mathcal{F}_{n+1}} \Sigma^{\mu_{n+1}}(A)$ : hence $\Sigma^{\mu_{n}}(A)<\mathrm{L} \Sigma^{\mu_{n+1}}(A)$ by Lemma 2.1.

In particular, Theorem 2.2, Corollary 4.7 and Theorem 5.3 implies that the degree-structure induced by any regular chain of reductions $\overrightarrow{\mathcal{F}}$ (i.e. by the preorder $\left.\leq_{\overrightarrow{\mathcal{F}}}\right)$ is Lipschitz-like.

\footnotetext{
${ }^{6}$ It is easy to check that each chain of reductions is equivalent either to a Borel-amenable set of reductions (if it has successor or uncountable rank) or to a regular chain of reductions with the same rank.

${ }^{7}$ For simplicity of notation, from now on we will systematically identify $\overrightarrow{\mathcal{F}}$ with $\bigcup_{n} \mathcal{F}_{n}$ when there is no possibility of misunderstanding.
} 


\section{BAIRE REDUCTIONS}

Let $\mathcal{B}_{\alpha}$ (for $\alpha<\omega_{1}$ ) denote the set of all Baire class $\alpha$ functions from $\mathbb{R}$ into itself, i.e. the set of all functions $f: \mathbb{R} \rightarrow \mathbb{R}$ such that $f^{-1}(U) \in \Sigma_{\alpha+1}^{0}$ for every open set $U$. Clearly $\mathrm{D}_{1}=\mathcal{B}_{0} \subseteq \mathcal{B}_{\alpha} \subseteq$ Bor for every $\alpha<\omega_{1}$, and $\mathcal{B}_{\mu} \subseteq \mathcal{B}_{\nu}$ if and only if $\mu \leq \nu$. Moreover it is well known that the Baire class $\alpha$ functions provides a stratification of Bor in $\omega_{1}$-many levels which is alternative to the one induced by $\boldsymbol{\Delta}_{\xi}^{0}$-functions, thus it is quite natural to try to study the reducibilities induced by the Baire class functions (of some level). Unfortunately, if $\alpha \neq 0$ then $\mathcal{B}_{\alpha}$ is not a set of reductions as it is not closed under composition: in fact, it is easy to check that if $f \in \mathcal{B}_{\mu}$ and $g \in \mathcal{B}_{\nu}$ then $g \circ f \in \mathcal{B}_{\mu+\nu}$ and, moreover, there are such an $f$ and $g$ for which $g \circ f \notin \mathcal{B}_{\eta}$ for any $\eta<\mu+\nu$. Nevertheless, we can exactly compute the closure under composition of $\mathcal{B}_{\alpha}$ by reversing the previous composition law, i.e. by showing that if $h \in \mathcal{B}_{\mu+\nu}$ then there are $f \in \mathcal{B}_{\mu}$ and $g \in \mathcal{B}_{\nu}$ such that $h=g \circ f$. To obtain this computation we will use Lemma 4.3 together with the following crucial fact (for simplicity of notation we will put $\boldsymbol{\Delta}_{0}^{0}=\boldsymbol{\Delta}_{1}^{0}$ ).

Lemma 6.1 $\left(\mathrm{ZF}+\mathrm{AC}_{\omega}(\mathbb{R})\right)$. For every nonzero $\mu, \nu<\omega_{1}$ with $\nu>1$ and every $\mathcal{C}=\left\{C_{n} \mid n \in \omega\right\} \subseteq \boldsymbol{\Delta}_{\mu+\nu}^{0}$ there is $\mathcal{B}=\left\{B_{m} \mid m \in \omega\right\} \subseteq \boldsymbol{\Delta}_{\mu+1}^{0}$ such that $\mathcal{C} \subseteq \boldsymbol{\Delta}_{\nu}^{0}\left(\tau^{\prime}\right)$ for every topology $\tau^{\prime}$ for which $\mathcal{B} \subseteq \boldsymbol{\Delta}_{1}^{0}\left(\tau^{\prime}\right)$.

Proof. Clearly we can assume that $\mathcal{C}$ is closed under complementation (if not simply adjoin $\neg C_{n}$ to $\mathcal{C}$ for every $n$ ). We will prove the lemma by induction on $\nu$, and the base of the induction and the successor case will be proved together. Assume $\nu=\eta+1$ (with $\eta \geq 1$ ): by definition there must be a collection $\mathcal{D}^{\prime}=\left\{D_{n, k}^{\prime} \mid n, k \in\right.$ $\omega\} \subseteq \boldsymbol{\Pi}_{\mu+\eta}^{0}$ such that $C_{n}=\bigcup_{k \in \omega} D_{n, k}^{\prime}$ for every $n$, and by definition again there must be some $\mathcal{D}=\left\{D_{n, k, i} \mid n, k, i \in \omega\right\} \subseteq \boldsymbol{\Delta}_{\mu+\eta}^{0}$ such that $D_{n, k}^{\prime}=\bigcap_{i \in \omega} D_{n, k, i}$ for every $n, k$. Put $\mathcal{B}=\mathcal{D}$ if $\eta=1$ or, in the other case, use the inductive hypothesis applied to $\mathcal{D}$ to find some countable $\mathcal{B} \subseteq \boldsymbol{\Delta}_{\mu+1}^{0}$ such that for every topology $\tau^{\prime}$ on $\mathbb{R}$ if $\mathcal{B} \subseteq \boldsymbol{\Delta}_{1}^{0}\left(\tau^{\prime}\right)$ then $\mathcal{D} \subseteq \boldsymbol{\Delta}_{\eta}^{0}\left(\tau^{\prime}\right)$. In both cases $\mathcal{D}^{\prime} \subseteq \boldsymbol{\Pi}_{\eta}^{0}\left(\tau^{\prime}\right)$ and $\mathcal{C} \subseteq \boldsymbol{\Delta}_{\eta+1}^{0}\left(\tau^{\prime}\right)$ (by closure under complementation of $\mathcal{C}$ ), hence we are done.

Now let $\nu$ be limit and let $\left\langle\nu_{i} \mid i \in \omega\right\rangle$ be any increasing sequence of ordinals cofinal in $\nu$ such that $\nu_{i}>1$ for every $i$. Since $\mathcal{C} \subseteq \Delta_{\mu+\nu}^{0}$ there must be some $\mathcal{D}=$ $\left\{D_{n, k} \mid n, k \in \omega\right\} \subseteq \boldsymbol{\Delta}_{<(\mu+\nu)}^{0}$ such that $C_{n}=\bigcup_{k \in \omega} D_{n, k}$. Put $\mathcal{D}_{i}=\left\{D_{n, k} \in \mathcal{D} \mid\right.$ $\left.D_{n, k} \in \boldsymbol{\Delta}_{\mu+\nu_{i}}^{0}\right\}$ for every $i$, so that $\mathcal{D}=\bigcup_{i \in \omega} \mathcal{D}_{i}$. Applying the inductive hypothesis to each $\mathcal{D}_{i}$ and using $\mathrm{AC}_{\omega}(\mathbb{R})$, we can find for every $i$ a collection $\mathcal{B}_{i} \subseteq \boldsymbol{\Delta}_{\mu+1}^{0}$ such that if $\tau^{\prime}$ is any topology on $\mathbb{R}$ for which $\mathcal{B}_{i} \subseteq \boldsymbol{\Delta}_{1}^{0}\left(\tau^{\prime}\right)$ then $\mathcal{D}_{i} \subseteq \boldsymbol{\Delta}_{\nu_{i}}^{0}\left(\tau^{\prime}\right)$. Put now $\mathcal{B}=\bigcup_{i \in \omega} \mathcal{B}_{i}$. Then $\mathcal{B} \subseteq \boldsymbol{\Delta}_{\mu+1}^{0}$ and if $\tau^{\prime}$ is such that $\mathcal{B} \subseteq \boldsymbol{\Delta}_{1}^{0}\left(\tau^{\prime}\right)$ then $\mathcal{D} \subseteq \boldsymbol{\Delta}_{<\nu}^{0}\left(\tau^{\prime}\right)$ and hence $\mathcal{C} \subseteq \boldsymbol{\Delta}_{\nu}^{0}\left(\tau^{\prime}\right)$.

Recall now the following classical fact: if $X$ is a zero-dimensional Polish space then there is a closed set $F \subseteq \mathbb{R}$ and an homeomorphism $H: F \rightarrow X$.

Proposition 6.2 (ZF $\left.+\mathrm{AC}_{\omega}(\mathbb{R})\right)$. Let $h: \mathbb{R} \rightarrow \mathbb{R}$ be in $\mathcal{B}_{\mu+\nu}$ (for some countable ordinals $\mu$ and $\nu)$. Then there are $f \in \mathcal{B}_{\mu}$ and $g \in \mathcal{B}_{\nu}$ such that $h=g \circ f$.

Proof. Let $\tau$ be the usual topology on $\mathbb{R}$. If $\mu=0$ or $\nu=0$ the result is trivial (simply take $f=\mathrm{id}$ and $g=h$ or, respectively, $f=h$ and $g=\mathrm{id}$ ). Hence we can assume $\mu, \nu>0$. Put $\mathcal{C}=\left\{h^{-1}\left(\mathbf{N}_{s}\right) \mid s \in<\omega \omega\right\} \subseteq \boldsymbol{\Delta}_{\mu+\nu+1}^{0}$. Let $\mathcal{B} \subseteq \boldsymbol{\Delta}_{\mu+1}^{0}$ be obtained as in the previous lemma, that is such that for any topology $\tau^{\prime}$ if $\mathcal{B} \subseteq \boldsymbol{\Delta}_{1}^{0}\left(\tau^{\prime}\right)$ then $\mathcal{C} \subseteq \boldsymbol{\Delta}_{\nu+1}^{0}\left(\tau^{\prime}\right)$. Apply Lemma 4.3 to $\mathcal{B}$ in order to obtain a zerodimensional Polish topology $\tau^{\prime}$ such that $\mathcal{B} \subseteq \boldsymbol{\Delta}_{1}^{0}\left(\tau^{\prime}\right)$ and let $F \subseteq \mathbb{R}$ be a closed set such that $H:(F, \tau) \rightarrow\left(\mathbb{R}, \tau^{\prime}\right)$ is an homeomorphism. Finally let $r: \mathbb{R} \rightarrow F$ be a retraction. Now put $g=h \circ H \circ r:(\mathbb{R}, \tau) \rightarrow(\mathbb{R}, \tau)$ and $f=H^{-1}:(\mathbb{R}, \tau) \rightarrow(\mathbb{R}, \tau)$. It is easy to check that $h:\left(\mathbb{R}, \tau^{\prime}\right) \rightarrow(\mathbb{R}, \tau)$ is of Baire class $\nu$, and thus also $g$ is of 
Baire class $\nu$. Moreover, since $H^{-1}:\left(\mathbb{R}, \tau^{\prime}\right) \rightarrow(F, \tau)$ is continuous and $\boldsymbol{\Delta}_{1}^{0}\left(\tau^{\prime}\right) \subseteq$ $\boldsymbol{\Delta}_{\mu+1}^{0}(\tau)$, we have that $f$ is of Baire class $\mu$. Thus we have only to prove that $g \circ f=h$. Since range $\left(H^{-1}\right)=F$, we have that $r\left(H^{-1}(x)\right)=\operatorname{id}\left(H^{-1}(x)\right)=H^{-1}(x)$ for every $x \in \mathbb{R}$. But then

$$
g \circ f(x)=h\left(H\left(r\left(H^{-1}(x)\right)\right)\right)=h\left(H\left(H^{-1}(x)\right)\right)=h(x) .
$$

Observe that the same statement is true if we replace $h$ with a $\boldsymbol{\Sigma}_{\mu+\nu}^{0}$-measurable function (with $\mu, \nu>1$ ) and we require that there are a $\boldsymbol{\Sigma}_{\mu}^{0}$-measurable function and a $\boldsymbol{\Sigma}_{\nu}^{0}$-measurable function whose composition gives $h$.

Remark 6.3. The previous proposition can be applied also to other Polish spaces $\mathscr{X}$ (clearly we can assume again that $\mu, \nu \neq 0$, otherwise the result is trivial). In fact the same argument shows that for every $h: \mathscr{X} \rightarrow \mathscr{X}$ of Baire class $\mu+\nu$ there are $f: \mathscr{X} \rightarrow \mathbb{R}$ of Baire class $\mu$ and $g: \mathbb{R} \rightarrow \mathscr{X}$ of Baire class $\nu$ such that $h=g \circ f$. Moreover, if we assume that $\mathscr{X}$ is (uncountable and) not $\mathbf{K}_{\sigma}$, the same result remains true also replacing $f$ and $g$ with two functions $f^{\prime}, g^{\prime}: \mathscr{X} \rightarrow \mathscr{X}$ of Baire class $\mu$ and $\nu$, respectively. In fact in this case there is a closed set $F$ of $\mathscr{X}$ which is homeomorphic to $\mathbb{R}$ via some function $H^{\prime}$, hence one can define $f^{\prime}=H^{\prime-1} \circ f$ and $g^{\prime}=\left(g \circ H^{\prime}\lceil F) \cup\left(f_{0}\lceil\mathscr{X} \backslash F)\right.\right.$, where $f$ and $g$ are obtained as in the previous proof and $f_{0}$ is any constant function, and check that they are still of the correct Baire class. Finally, this last version of Proposition 6.2 can be further extended to every uncountable Polish space $\mathscr{X}$ if we assume $\nu \neq 1$ : in fact in this case we can use the fact that every zero-dimensional Polish space is homeomorphic to some $\mathbf{G}_{\delta}$ subspace of the Cantor space ${ }^{\omega} 2$, which is in turn homeomorphic to a closed subset of $\mathscr{X}$. Therefore any zero-dimensional Polish space is homeomorphic to a $\mathbf{G}_{\delta}$ set $G$ of $\mathscr{X}$ via some function $H^{\prime}$, and we can define $f^{\prime}$ and $g^{\prime}$ as above but replacing $F$ with $G$.

Theorem 6.4 $\left(\mathrm{ZF}+\mathrm{AC}_{\omega}(\mathbb{R})\right)$. Let $\alpha$ be a nonzero countable ordinal. Then the closure under composition of $\mathcal{B}_{\alpha}$ is exactly $\bigcup_{\mu<\xi} \mathcal{B}_{\mu}$, where $\xi=\alpha \cdot \omega$ is the least additively closed ordinal above $\alpha$.

Proof. One direction is trivial (since the composition of $n$ Baire class $\alpha$ functions is in $\mathcal{B}_{\alpha \cdot n}$ ). Suppose now that $h$ belongs to $\mathcal{B}_{\alpha \cdot n}$ for some $1 \leq n \in \omega$. We will prove by induction on $n$ that $h$ belongs to the closure under composition of $\mathcal{B}_{\alpha}$. If $n=1$ there is nothing to prove, while if $n=m+1$ (for some $m \geq 1$ ) then $h \in \mathcal{B}_{\alpha \cdot m+\alpha}$ and we can apply Proposition 6.2 to get $f \in \mathcal{B}_{\alpha \cdot m}$ and $g \in \mathcal{B}_{\alpha}$ such that $h=g \circ f$. Applying now the inductive hypothesis to $f$ we get the result.

By the previous theorem, we are naturally led to take any countable additively closed ordinal $\xi$ (recall that $\xi$ is additively closed if and only if either $\xi=0,1$ or $\xi=\omega^{\mu}$ for some ordinal $\mu$ ) and study the degree structure induced by

$$
\mathrm{B}_{\xi}=\mathcal{B}_{<\xi}=\bigcup_{\mu<\xi} \mathcal{B}_{\mu}
$$

(by the rule of composition above, $\mathrm{B}_{\xi}$ is closed under composition and hence it is a Borel set of reductions). Since it is straightforward to check that $\mathrm{B}_{\xi}$ is a good Borel reducibility (and therefore has the SDP), and that $\Delta_{\mathrm{B}_{\xi}}=\boldsymbol{\Delta}_{<\xi}^{0}$ (which in particular implies that $\mathrm{B}_{\xi}$ is of type III), we can apply Theorem 4.8 to get that $\mathrm{B}_{\xi}$ is equivalent to any (regular) chain of reductions of rank $\xi$, and thus induces the same degree-structure.

This equivalence is non trivial (at least for $\xi=\omega$ ): in fact we will show that $\mathrm{B}_{\omega}$ is not contained in $\bigcup_{n \in \omega} D_{n}$ by proving that there is a Baire class 1 function which is not in $\mathrm{D}_{n}$ for any $n \in \omega$ (this discussion will also cover the missing proofs about the Pawlikowski function in [8]). First let us recall the definition of the Pawlikowski 
function $P$ from [10]. Let $\omega+1$ have the order topology and consider the space $\omega(\omega+1)$ endowed with the corresponding product topology. It is easy to check that ${ }^{\omega}(\omega+1)$ is perfect, zero-dimensional and compact, hence it is homeomorphic to the Cantor space ${ }^{\omega} 2$. Let $\gamma: \omega+1 \rightarrow \omega$ be the bijection defined by $\gamma(\omega)=0$ and $\gamma(n)=n+1$ for any $n \in \omega$, and define $P:{ }^{\omega}(\omega+1) \rightarrow \mathbb{R}$ using $\gamma$ coordinatewise, i.e. putting $P(x)=\langle\gamma(x(n)) \mid n \in \omega\rangle$. Define also (again coordinatewise) a "partial" function

$$
\hat{\gamma}:<\omega(\omega+1) \rightarrow{ }^{<\omega} \omega: s \mapsto\langle\gamma(s(i)) \mid i<\ln (s)\rangle,
$$

and note that both $P$ and $\hat{\gamma}$ are bijection between the corresponding spaces.

Given $\tau \in{ }^{<\omega}(\omega+1)$, consider the set $C_{\tau}=\left\{x \in{ }^{\omega}(\omega+1) \mid \tau \subseteq x\right\}$ : by simple arguments, it turns out that $C_{\tau}$ is always a closed set, has empty interior if and only if there is some $i<\operatorname{lh}(\tau)$ such that $\tau(i)=\omega$, and is also open (hence a clopen set) if and only if $\tau(i) \neq \omega$ for every $i<\operatorname{lh}(\tau)$. In particular, this implies that for every $n \in \omega$ the set $K_{n}=\left\{x \in{ }^{\omega}(\omega+1) \mid x(n)=\omega\right\}$ is a closed set with empty interior and hence a nowhere dense proper closed set (from this fact one can also derive that ${ }^{\omega} \omega$ is a proper $\mathbf{G}_{\delta}$ subset of ${ }^{\omega}(\omega+1)$ which is also comeager and dense in it).

Lemma 6.5 $\left(\mathrm{ZF}+\mathrm{AC}_{\omega}(\mathbb{R})\right)$. Let $\mathscr{X}$ be any zero-dimensional space. Let $\alpha<\omega_{1}$ be a nonzero ordinal and let $\left\langle\alpha_{n} \mid n \in \omega\right\rangle$ be an increasing sequence of ordinals smaller than $\alpha$ and cofinal in it. For every family of sets $\left\{P_{n} \subseteq \omega \mathscr{X} \mid n \in \omega\right\}$ such that $P_{n}$ is $\Pi_{\alpha_{n}}^{0}$-complete (for every $n \in \omega$ ), the set $S \subseteq{ }^{\omega} \mathscr{X}$ defined by

$$
S=\left\{x \in{ }^{\omega} \mathscr{X} \mid \exists n\left(\pi_{n}^{\mathscr{X}}(x) \in P_{n}\right)\right\}
$$

is a $\boldsymbol{\Sigma}_{\alpha}^{0}$-complete set. In particular, if $P \subseteq \omega \mathscr{X}$ is a $\boldsymbol{\Pi}_{\alpha}^{0}$-complete set then $S=$ $\left\{x \in{ }^{\omega} \mathscr{X} \mid \exists n\left(\pi_{n}^{\mathscr{X}}(x) \in P\right)\right\}$ is a $\boldsymbol{\Sigma}_{\alpha+1}^{0}$-complete set.

Proof. Clearly $S \in \boldsymbol{\Sigma}_{\alpha}^{0}$. Let now $Q$ be any set in $\boldsymbol{\Sigma}_{\alpha}^{0}$ : by definition there is an increasing sequence $\left\langle\beta_{k} \mid k \in \omega\right\rangle$ of ordinals smaller than $\alpha$ such that $Q=\bigcup_{k} R_{k}$ for some sets $R_{k} \in \Pi_{\beta_{k}}^{0}$. Since the sequence $\left\langle\alpha_{n} \mid n \in \omega\right\rangle$ is increasing and cofinal in $\alpha$ we can find a subsequence $\left\langle\alpha_{n_{k}} \mid k \in \omega\right\rangle$ such that $\beta_{k} \leq \alpha_{n_{k}}$ for any $k \in \omega$. Moreover, since every $P_{n}$ is $\Pi_{\alpha_{n}}^{0}$-complete we can choose a sequence of points $\left\langle x_{n} \mid n \in \omega\right\rangle$ such that $x_{n} \notin P_{n}$ for every $n \in \omega$. Now define a sequence of continuous functions $\left\langle f_{n} \mid n \in \omega\right\rangle$ by letting $f_{n_{k}}$ be any continuous reduction of $R_{k}$ in $P_{n_{k}}$ (which exists since $R_{k} \in \mathbf{\Pi}_{\beta_{k}}^{0} \subseteq \boldsymbol{\Pi}_{\alpha_{n_{k}}}^{0}$ ), and $f_{n}$ be the constant function with value $x_{n}$ if there is no $k \in \omega$ such that $n=n_{k}$. Finally, put $f=\bigotimes_{n}^{\mathscr{X}} f_{n}$. Clearly $f$ is continuous and it is not hard to check that it reduces $Q$ to $S$, i.e. that $x \in Q \Longleftrightarrow f(x) \in S$ for every $x \in{ }^{\omega} \mathscr{X}$.

Now we are ready to prove the following proposition which gives the exact complexity of $P$.

Proposition 6.6 $\left(\mathrm{ZF}+\mathrm{AC}_{\omega}(\mathbb{R})\right)$. The function $P$ is of Baire class 1 and is in $\mathrm{D}_{\omega}$ but not in $\mathrm{D}_{n}$ (for any nonzero $n \in \omega$ ).

Proof. Since $P^{-1}\left(\mathbf{N}_{s}\right)=C_{\hat{\gamma}^{-1}(s)}$ for every $s \in{ }^{<\omega} \omega$, we have that $P^{-1}(U)$ is the union of countably many closed sets for any open set $U \subseteq \mathbb{R}$ : hence $P$ is of Baire class 1 (this also implies that $P \in \mathrm{D}_{\omega}$ since every Baire class $n$ function is in $\mathrm{D}_{\omega}$ ). It remains only to prove that $P$ is not in $\mathrm{D}_{n}$ for any $n \in \omega$. First define

$$
\begin{aligned}
S_{1} & =\{x \in \mathbb{R} \mid \exists n(x(n)=0)\} \\
S_{n+1} & =\left\{x \in \mathbb{R} \mid \exists n\left(\pi_{n}(x) \notin S_{n}\right\}\right.
\end{aligned}
$$

for $n \geq 1$. One can inductively check that $S_{n} \subseteq \mathbb{R}$ is a $\boldsymbol{\Sigma}_{n}^{0}$ set, and that $P^{-1}\left(S_{n}\right) \subseteq$ $\omega(\omega+1)$ is a complete (and hence also proper) $\boldsymbol{\Sigma}_{n+1}^{0}$ set (use Lemma 6.5 for the 
inductive step). Passing to the complements, we have that $\neg S_{n} \in \boldsymbol{\Pi}_{n}^{0} \subseteq \boldsymbol{\Delta}_{n+1}^{0}$ but $P^{-1}\left(\neg S_{n}\right) \notin \boldsymbol{\Delta}_{n+1}^{0}$, i.e. $P$ is not a $\boldsymbol{\Delta}_{n+1}^{0}$-function.

The function $P$ is defined from ${ }^{\omega}(\omega+1)$ to $\mathbb{R}$, while we are interested in functions from $\mathbb{R}$ into itself. Nevertheless it is easy to see how to obtained from $P$ a function $\hat{P}: \mathbb{R} \rightarrow \mathbb{R}$ with the same complexity. Let $h:{ }^{\omega}(\omega+1) \rightarrow{ }^{\omega} 2$ be any homeomorphism between ${ }^{\omega}(\omega+1)$ and the Cantor space ${ }^{\omega} 2$ (which is a closed subspace of $\mathbb{R})$. Define $\hat{P}: \mathbb{R} \rightarrow \mathbb{R}$ by letting $\hat{P}(x)=P\left(h^{-1}(x)\right)$ if $x \in{ }^{\omega_{2}}$ and $\hat{P}(x)=\overrightarrow{0}$ otherwise. Following [10], for every $f: X_{1} \rightarrow Y_{1}$ and $g: X_{2} \rightarrow Y_{2}$ put $f \sqsubseteq g$ just in case there are two embeddings $\varphi: X_{1} \rightarrow X_{2}$ and $\psi: f\left(X_{1}\right) \rightarrow Y_{2}$ such that $\psi \circ f=g \circ \varphi$. Clearly, if $f \sqsubseteq g$ and $g$ is a $\boldsymbol{\Delta}_{\xi}^{0}$-function (respectively, a Baire class $\xi$ function) then also $f$ is a $\boldsymbol{\Delta}_{\xi}^{0}$-function (respectively a Baire class $\xi$ function), and therefore if $f$ is not a $\boldsymbol{\Delta}_{\xi}^{0}$-function (resp. a Baire class $\xi$ function) then neither $g$ is a $\boldsymbol{\Delta}_{\xi}^{0}$-function (resp. a Baire class $\xi$ function). Since it is not hard to prove that $\hat{P}$ is still a Baire class 1 function and that $h$ and the identity function witness $P \sqsubseteq \hat{P}$, we have that $\hat{P} \in \mathrm{D}_{\omega}$ but $\hat{P} \notin \mathrm{D}_{n}$ for any $n \in \omega$, hence we are done.

\section{Comparing hierarchies of DEgREeS}

All sets of functions considered in this section are assumed to be good Borel reducibilities. Let $\mathcal{G}_{\mu}$ denote an arbitrary good Borel set of reductions with $\Delta_{\mathcal{G}_{\mu}}=\boldsymbol{\Delta}_{\mu}^{0}$ (in particular $\mathcal{G}_{\mu}$ is always of type II). To clarify the relationship between (the degree-structures induced by) different good Borel reducibilities, note that each $\mathcal{F}$ of type I induces the finest possible hierarchy (in particular finer than the hierarchy induced by any $\mathcal{G}_{1}$, which is in some sense the next "level of reducibility"), and each $\mathcal{H}$ of type III with $\Delta_{\mathcal{H}}=\Delta_{<\xi}^{0}$ (for $\xi$ a countable limit ordinal) induces an hierarchy of degrees which is coarser than the hierarchy of the $\mathcal{G}_{\mu}$-degrees (for any $\mu<\xi$ ), and finer than the hierarchy of the $\mathcal{G}_{\xi}$-degrees. Finally Bor, and the sets of reductions with the same characteristic set, gives the coarsest hierarchy. By part vi) of Theorem 2.2, it is clear that for an $\mathcal{F}$-hierarchy being coarser than the $\mathcal{F}^{\prime}$-hierarchy amount to the fact that the $\mathcal{F}$-selfdual degrees are obtained gluing together many $\mathcal{F}^{\prime}$-degrees: therefore to understand how the $\mathcal{F}$-structure can be obtained from the finer ones we must describe how each $\mathcal{F}$-selfdual degree is constructed.

The first case, that is when we want to compare the $\mathcal{F}$-hierarchy (for $\mathcal{F}$ of type I) with the $\mathcal{G}_{1}$-hierarchy, is clearly solved by the Steel-Van Wesep Theorem, which says that $A \leq_{\mathrm{W}} \neg A \Longleftrightarrow A \leq_{\mathrm{L}} \neg A$ : in fact since $\mathrm{L} \subseteq \mathcal{F} \subseteq \mathrm{W} \simeq \mathcal{G}_{1}$ it must be the case that $A \leq_{\mathcal{G}_{1}} \neg A$ if and only if $A \leq_{\mathcal{F}} \neg A$, and as $s_{\text {Lip }}(A) \leq_{\mathrm{W}} A$ we get that each $\mathcal{G}_{1}$-selfdual degree is exactly the union of a (maximal) $\omega_{1}$-chain of consecutive $\mathcal{F}$-selfdual degrees.

Now consider $\mathcal{H}$ of type III as above: as $\mathcal{H} \simeq \bigcup_{\mu<\xi} \mathcal{G}_{\mu}$, it is clear that if $A \leq \mathcal{H} \neg A$ then $[A]_{\mathcal{H}}=\bigcup_{\mu<\xi}[A]_{\mathcal{G}_{\mu}}$. Therefore the $\mathcal{H}$-hierarchy is the minimal degree-structure which is refined by all the $\mathcal{G}_{\mu}$-structures.

Finally, to compare the $\mathcal{H}$-hierarchy with the $\mathcal{G}_{\xi}$-hierarchy, for $\overrightarrow{\mathcal{F}}$ a regular chain of reductions first define ${ }^{8} s_{\xi}^{\alpha}[B]_{\overrightarrow{\mathcal{F}}}$ (for $B \subseteq \mathbb{R}$ and $1 \leq \alpha<\omega_{1}$ ) by letting $s_{\xi}^{1}[B]_{\overrightarrow{\mathcal{F}}}=$ $[B]_{\overrightarrow{\mathcal{F}}}, s_{\xi}^{\alpha}[B]_{\overrightarrow{\mathcal{F}}}=\left[\bigoplus_{n} C_{n}\right]_{\overrightarrow{\mathcal{F}}}$ (where $C_{n} \in s_{\xi}^{\alpha_{n}}[B]_{\overrightarrow{\mathcal{F}}}$ and the $\alpha_{n}$ 's are increasing and cofinal in $\alpha$ ) if $\alpha$ is limit, and $s_{\xi}^{\alpha}[B]_{\overrightarrow{\mathcal{F}}}=\left[s_{\overrightarrow{\mathcal{F}}}(C)\right]_{\overrightarrow{\mathcal{F}}}$ (where $C \in s_{\xi}^{\alpha^{\prime}}[B]_{\overrightarrow{\mathcal{F}}}$ ) if $\alpha=\alpha^{\prime}+1$. (Note that if $B \leq_{\overrightarrow{\mathcal{F}}} \neg B$ then the $s_{\xi}^{\alpha}[B]_{\overrightarrow{\mathcal{F}}}$ 's are exactly the $\omega_{1}$-chain of consective $\overrightarrow{\mathcal{F}}$ selfdual degrees which follows $[B]_{\overrightarrow{\mathcal{F}}}$.) Moreover, given a pointclass $\Gamma$ and a nonzero

\footnotetext{
${ }^{8}$ We must define the $\omega_{1}$-chain on the $\overrightarrow{\mathcal{F}}$-degrees (rather than on sets) because the Perfect Set Property, which already follows from SLO ${ }^{\mathrm{L}}$, forbids the possibility of having an $\omega_{1}$-chain of sets of bounded Borel rank.
} 
ordinal $\mu<\omega_{1}$, define

$$
\mathrm{PU}_{\mu}(\Gamma)=\left\{\bigcup_{n}\left(A_{n} \cap D_{n}\right) \mid A_{n} \in \Gamma \text { and }\left\langle D_{n} \mid n \in \omega\right\rangle \text { is a } \boldsymbol{\Delta}_{\mu}^{0} \text {-partition of } \mathbb{R}\right\}
$$

and

$$
\mathrm{SU}_{\mu}(\Gamma)=\left\{\bigcup_{n}\left(A_{n} \cap D_{n}\right) \mid A_{n} \in \Gamma, D_{n} \in \boldsymbol{\Delta}_{\mu}^{0} \text { and } D_{n} \cap D_{m}=\emptyset \text { if } n \neq m\right\} .
$$

Finally, for $\mu$ limit and $\mu_{n}$ 's (strictly) increasing and cofinal in $\mu$ define $\mathrm{SU}_{<\mu, \alpha}(\Gamma)$ by the following induction on $\alpha<\omega_{1}$ (note the definition is independent from the choice of the $\mu_{n}$ 's):

$$
\mathrm{SU}_{<\mu, \alpha}(\Gamma)= \begin{cases}\Gamma & \text { if } \alpha=0 \\ \bigcup_{n} \mathrm{SU}_{\mu_{n}}\left(\bigcup_{\alpha^{\prime}<\alpha} \mathrm{SU}_{<\mu, \alpha^{\prime}}(\Gamma)\right) & \text { if } \alpha>0\end{cases}
$$

Proposition 7.1 $\left(\mathrm{ZF}+\mathrm{AC}_{\omega}(\mathbb{R})\right)$. Let $\overrightarrow{\mathcal{F}}$ be a regular chain of reductions of rank $\xi$ (for $\xi$ a countable limit ordinal). For $A, B \subseteq \mathbb{R}, A \leq_{\mathrm{D}_{\xi}^{\mathrm{w}}} B$ if and only if $A \leq \leq_{\mathrm{w}} C$ for some $C \in s_{\xi}^{\alpha}[B]_{\overrightarrow{\mathcal{F}}}$ and $\alpha<\omega_{1}$.

Proof. Let $\boldsymbol{\Gamma}=\boldsymbol{\Gamma}(B)=\{D \subseteq \mathbb{R} \mid D \leq \mathrm{w} B\}$ be the boldface pointclass generated by $B$. It is immediate to check that $A \leq_{\mathrm{D}_{\xi}^{w}} B \Longleftrightarrow A \in \mathrm{PU}_{\xi}(\boldsymbol{\Gamma})$. By Theorem E.4 of chapter IV of Wadge's [13], $\mathrm{PU}_{\xi}(\boldsymbol{\Gamma})=\bigcup_{\alpha<\omega_{1}} \mathrm{SU}_{<\xi, \alpha}(\boldsymbol{\Gamma})$, so let $\alpha$ be smallest such that $A \in \mathrm{SU}_{<\xi, \alpha}(\boldsymbol{\Gamma})$. We will prove by induction on $\alpha$ that $A \leq_{\mathrm{W}} C$ for some $C \in s_{\xi}^{\alpha+1}[B]_{\overrightarrow{\mathcal{F}}}$. If $\alpha=0$, then $A \in \mathrm{SU}_{<\xi, 0}(\boldsymbol{\Gamma})=\boldsymbol{\Gamma}$ and therefore $A \leq \mathrm{w} B$ (by definition of $\boldsymbol{\Gamma}$ ) and obviously $B \in[B]_{\overrightarrow{\mathcal{F}}}=s_{\xi}^{1}[B]_{\overrightarrow{\mathcal{F}}}$. Now assume $\alpha>0$, and let $n$ be such that $A \in \mathrm{SU}_{\mu_{n}}\left(\bigcup_{\alpha^{\prime}<\alpha} \mathrm{SU}_{<\mu, \alpha^{\prime}}(\boldsymbol{\Gamma})\right)$, so that $A=\bigcup_{m}\left(A_{m} \cap D_{m}\right)$ where $D_{m} \in \boldsymbol{\Delta}_{\mu_{n}}^{0}, D_{m} \cap D_{m^{\prime}}=\emptyset$ if $m \neq m^{\prime}$, and $A_{m} \in \mathrm{SU}_{<\mu, \alpha_{m}}(\boldsymbol{\Gamma})$ for some $\alpha_{m}<\alpha$ (depending on $m$ ). By inductive hypothesis, $A_{m} \leq \mathrm{w} C_{m}$ for some $C_{m} \in s_{\xi}^{\alpha_{m}+1}[B]_{\overrightarrow{\mathcal{F}}}$, and therefore $A_{m} \leq \mathrm{w} \bigoplus_{m} C_{m}$ for every $m$. Moreover $\bigoplus_{m} C_{m} \in s_{\xi}^{\alpha}[B]_{\overrightarrow{\mathcal{F}}}$ as $\alpha=$ $\sup \left\{\alpha_{m}+1 \mid m \in \omega\right\}$ by its minimality.

Claim 7.1.1. $A \leq \mathrm{W} \Sigma^{\mu_{n}+1}\left(\bigoplus_{m} C_{m}\right)$.

Proof of Claim. Let $\left\langle\mu_{n} \mid n \in \omega\right\rangle$ be the type of $\overrightarrow{\mathcal{F}}, P$ be the complete $\Pi_{\mu_{n}}^{0}$-set used to define the operator $\Sigma^{\mu_{n}+1}$, and $f_{m}$ be continuous functions such that $f_{0}$ reduces $\neg \bigcup_{m} D_{m}$ to $P, f_{2(m+1)}$ reduces $D_{m}$ to $P, f_{1}$ is constant with value $y \notin \bigoplus_{m} C_{m}$, and $f_{2 m+3}$ is a reduction of $A_{m}$ to $\bigoplus_{m} C_{m}$ : it is easy to check that $\bigotimes_{m} f_{m}$ reduces $A$ to $\Sigma^{\mu_{n}+1}\left(\bigoplus_{m} C_{m}\right)$ as required.

Claim

Since $\mu_{n}<\mu_{n}+1 \leq \mu_{n+1}$, we get $\Sigma^{\mu_{n}+1}\left(\bigoplus_{m} C_{m}\right) \leq \mathrm{w} \quad \Sigma^{\mu_{n+1}}\left(\bigoplus_{m} C_{m}\right) \leq \mathrm{w}$ $s_{\overrightarrow{\mathcal{F}}}\left(\bigoplus_{m} C_{m}\right) \in s_{\xi}^{\alpha+1}[B]_{\overrightarrow{\mathcal{F}}}$ and hence we are done.

As a corollary of Proposition 7.1 one gets a Steel-Van Wesep-style theorem for higher levels.

Theorem 7.2. Let $\mathcal{G}_{\xi}$ be as above and $\overrightarrow{\mathcal{F}}$ be a regular chain of reductions of rank $\xi$. Then $A \leq_{\mathcal{G}_{\xi}} \neg A$ if and only if $A \leq_{\overrightarrow{\mathcal{F}}} \neg A$. In particular, $A \leq_{\mathrm{D}_{\xi}} \neg A$ implies that $A \leq_{\mathrm{D}_{\mu}} \neg A$ for some $\mu<\xi$.

Proof. One direction is easy, as $\bigcup_{\mu<\xi} \mathrm{D}_{\mu} \subseteq \mathrm{D}_{\xi} \simeq \mathcal{G}_{\xi}$. For the other direction let $B$ be $\mathrm{L}$-minimal in $[A]_{\mathcal{G}_{\xi}}$, so that $B \leq_{\mathrm{L}} \neg B$. As $\mathcal{G}_{\xi} \simeq \mathrm{D}_{\xi}^{\mathrm{W}}$, apply Proposition 7.1 and let $\alpha$ be minimal such that $A \leq \mathrm{w} C$ for some $C \in s_{\xi}^{\alpha}[B]_{\overrightarrow{\mathcal{F}}}$. Since $A<_{\overrightarrow{\mathcal{F}}} C$ contradicts the minimality of $\alpha$, we must have $C \leq_{\overrightarrow{\mathcal{F}}} A$ and therefore $A \equiv_{\overrightarrow{\mathcal{F}}} C$ : but as $C$ is $\overrightarrow{\mathcal{F}}$-selfdual (since $B$ is) we get $A \leq_{\overrightarrow{\mathcal{F}}} \neg A$ as desired. 
All this discussion solves the problem of comparing the $\mathcal{H}$-hierarchy with the $\mathcal{G}_{\xi}$-hierarchy: using the fact that $\mathcal{G}_{\xi} \simeq \mathrm{D}_{\xi}^{\mathrm{W}}, \mathcal{H} \simeq \overrightarrow{\mathcal{F}}$ (where $\overrightarrow{\mathcal{F}}$ is any regular chain of rank $\xi$ ), and $s_{\overrightarrow{\mathcal{F}}}(A) \leq_{\mathrm{D}_{\xi}^{\mathrm{w}}} A$, we get that each $\mathcal{G}_{\xi}$-selfdual degree is exactly the union of a (maximal) $\omega_{1}$-chain of consecutive $\mathcal{H}$-selfdual degrees.

\section{Appendix: Some Alternative Proofs of the SDP}

In many of the concrete examples, one can directly prove (in a simpler way) that a Borel set of reductions $\mathcal{F}$ has the SDP using the fact that $\operatorname{Lip}(2) \subseteq \mathcal{F}$ and applying Proposition 4.2. For instance, if $\mathcal{F}=\operatorname{Lip}$ or $\mathcal{F}=$ UCont, given a set $A \leq_{\mathcal{F}} \neg A$ which is L-minimal in its $\mathcal{F}$-degree we can use the fact that $A \leq \mathrm{W} \neg A$ (since Lip $\subseteq$ UCont $\subseteq \mathrm{W}$ ) and then apply the Steel-Van Wesep Theorem to get $A \leq \mathrm{L} \neg A$.

In the case of a regular chains of reductions $\overrightarrow{\mathcal{F}}=\left\langle\mathcal{F}_{n} \mid n \in \omega\right\rangle$, we can prove that if $A \leq_{\overrightarrow{\mathcal{F}}} \neg A$ and $B$ is L-minimal in $[A]_{\overrightarrow{\mathcal{F}}}$ then $B \leq_{\mathrm{L}} \neg B$ as follows: let $n_{0}$ be minimal such that $A \leq \mathcal{F}_{n_{0}} \neg A$, so that $A \leq_{\mathcal{F}_{m}} \neg A$ for every $m \geq n_{0}$. Moreover, for every $m \geq n_{0}$ let $B_{m}$ be L-minimal in $[A]_{\mathcal{F}_{m}}$ (so that $B_{m} \leq_{\mathrm{L}} \neg B_{m}$ by Corollary 5.4 in [8]), and note that if $0 \leq k \leq m$ then $B_{m} \leq_{\mathrm{L}} C$ for every $C \in[A]_{\mathcal{F}_{k}}$ because $\overrightarrow{\mathcal{F}}$ is regular (in particular, if $n_{0} \leq k \leq m$ then $B_{m} \leq_{\mathrm{L}} B_{k}$ ). Since $\leq_{\mathrm{L}}$ is well-founded, there must be some $n_{1} \geq n_{0}$ such that $B_{m} \equiv_{\mathrm{L}} B_{n_{1}}$ for every $m \geq n_{1}$. Put $B^{\prime}=B_{n_{1}}$ : then $B^{\prime} \leq_{\mathrm{L}} \neg B^{\prime}$ and $B^{\prime}$ is easily seen to be $\mathrm{L}$-minimal in $[A]_{\overrightarrow{\mathcal{F}}}$, so that $B^{\prime} \equiv_{\mathrm{L}} B$ and we are done.

However, Baire reductions $\mathrm{B}_{\xi}$ are perhaps the most interesting case.

Lemma $\left(\mathrm{ZF}+\mathrm{AC}_{\omega}(\mathbb{R})\right)$. Let $A, B \subseteq \mathbb{R}$ be such that $A \leq_{\mathrm{B}_{\xi}} B$. Then there is some $C \equiv_{\mathrm{B}_{\xi}}$ A such that $C \leq_{\mathrm{L}} A$ and $C \leq_{\mathrm{L}} B$.

Proof. It is enough to prove that there is some $A^{\prime} \equiv_{\mathrm{B}_{\xi}} A$ such that $A^{\prime} \leq \mathrm{w} A$ and $A^{\prime} \leq \mathrm{w} B$ : then applying twice Lemma 19 of [1] we get the desired $C$ as in the proof of Lemma 8 in [4]. Let $\mu<\xi$ and $f \in \mathcal{B}_{\mu}$ be such that $A=f^{-1}(B)$. Applying Lemma 4.3 to the family $\left\{f^{-1}\left(\mathbf{N}_{s}\right) \mid s \in{ }^{<\omega} \omega\right\} \subseteq \boldsymbol{\Delta}_{\mu+1}^{0}$, we get a new zero-dimensional Polish topology $\tau^{\prime} \supseteq \tau$ on $\mathbb{R}$ such that $f:\left(\mathbb{R}, \tau^{\prime}\right) \rightarrow(\mathbb{R}, \tau)$ is continuous and $\boldsymbol{\Sigma}_{1}^{0}\left(\tau^{\prime}\right) \subseteq \boldsymbol{\Sigma}_{\mu+1}^{0}(\tau)$. Let $H:(F, \tau) \rightarrow\left(\mathbb{R}, \tau^{\prime}\right)$ be an homeomorphism between a closed set $F \subseteq \mathbb{R}$ and $\mathbb{R}$ endowed with the new topology, and let $r: \mathbb{R} \rightarrow F$ be a retraction on $F$. Finally, put $A^{\prime}=(H \circ r)^{-1}(A)$ : since $H^{-1}:(\mathbb{R}, \tau) \rightarrow(\mathbb{R}, \tau)$ is of Baire class $\mu$ we get $A \leq_{\mathrm{B}_{\xi}} A^{\prime}$, and since $H \circ r$ and $f \circ H \circ r$ are clearly continuous function from $(\mathbb{R}, \tau)$ to $(\mathbb{R}, \tau)$ which witness $A^{\prime} \leq \mathrm{w} A$ and $A^{\prime} \leq \mathrm{w} B$, respectively, $A^{\prime}$ is as required.

Let now $B$ be L-minimal in $[A]_{\mathrm{B}_{\xi}}$, where $A \leq_{\mathrm{B}_{\xi}} \neg A$. Since $B \leq_{\mathrm{B}_{\xi}} \neg B$, we can apply the previous lemma to get $C \equiv_{\mathrm{B}_{\xi}} B$ such that $C \leq_{\mathrm{L}} B$ and $C \leq_{\mathrm{L}} \neg B$. By minimality of $B$, we must have $B \equiv \mathrm{L} C$ and hence $B \leq_{\mathrm{L}} \neg B$.

\section{REFERENCES}

[1] Alessandro Andretta, Equivalence between Wadge and Lipschitz determinacy, Annals of Pure and Applied Logic, 123, 2003, 163-192.

[2] Alessandro Andretta, More on Wadge determinacy, Annals of Pure and Applied Logic, 144, $2006,2-32$.

[3] Alessandro Andretta, The SLO principle and the Wadge hierarchy, Foundations of the formal sciences V, Stud. Log. (Lond.), 11, 2007, 1-38.

[4] Alessandro Andretta and Donald A. Martin, Borel-Wadge degrees, Fundamenta Mathematicae, 177, 2003, 175-192.

[5] Jacques Duparc, A hierarchy of deterministic context-free $\omega$-languages, Theoretical Computer Science, 290, 2003, 1253-1300.

[6] Alexander S. Kechris, Classical Descriptive Set Theory, number 156 in Graduate Text in Mathematics. Springer-Verlag, Heidelberg, New York, 1995. 
[7] Luca Motto Ros, A new characterization of Baire class 1 functions, Real Analysis Exchange, 34 (1), 2008/2009, 29-48.

[8] Luca Motto Ros, Borel-amenable reducibilities for sets of reals, Journal of Symbolic Logic, 74 (1), 2009, 27-49.

[9] Luca Motto Ros, General Reducibilities for Sets of Reals, Ph.D. thesis, Polytechnic of Turin, Italy, 2007.

[10] Slawomir Solecki, Decomposing Borel sets and functions and the structure of Baire class 1 functions, Journal of the American Mathematical Society, 11 (3), 1998, 521-550.

[11] Robert M. Solovay, The independence of DC from AD, in Alexander S. Kechris and Yiannis N. Moschovakis, editors, Cabal Seminar 76-77, number 689 in Lecture Notes in Mathematics. Springer-Verlag, 1978.

[12] Robert A. Van Wesep, Wadge degrees and descriptive set theory, in Alexander S. Kechris and Yiannis N. Moschovakis, editors, Cabal Seminar 76-77, number 689 in Lecture Notes in Mathematics. Springer-Verlag, 1978.

[13] William W. Wadge, Reducibility and Determinateness on the Baire Space, Ph.D. thesis, University of California, Berkeley, 1983.

Kurt Gödel Research Center for Mathematical Logic, University of Vienna, Währinger Strasse 25, A-1090 Vienna, Austria

E-mail address: luca.mottoros@libero.it 